\title{
Bounded Conjunctive Queries
}

\author{
Wenfei Fan $^{1,2}$ \\ Yang $\mathrm{CaO}^{1,2}$
${ }^{1}$ University of Edinburgh \\ ${ }^{2}$ RCBD and SKLSDE Lab, Beihang University \\ \{Y.Cao-17@sms, wenfei@inf\}.ed.ac.uk \\ woty@act.buaa.edu.cn
}

\author{
Wenyuan $\mathrm{Yu}^{3}$ \\ ${ }^{3}$ Facebook Inc. \\ wyu@fb.com
}

\begin{abstract}
A query $Q$ is said to be effectively bounded if for all datasets $D$, there exists a subset $D_{Q}$ of $D$ such that $Q(D)=Q\left(D_{Q}\right)$, and the size of $D_{Q}$ and time for fetching $D_{Q}$ are independent of the size of $D$. The need for studying such queries is evident, since it allows us to compute $Q(D)$ by accessing a bounded dataset $D_{Q}$, regardless of how big $D$ is. This paper investigates effectively bounded conjunctive queries (SPC) under an access schema $\mathcal{A}$, which specifies indices and cardinality constraints commonly used. We provide characterizations (sufficient and necessary conditions) for determining whether an SPC query $Q$ is effectively bounded under $\mathcal{A}$. We study several problems for deciding whether $Q$ is bounded, and if not, for identifying a minimum set of parameters of $Q$ to instantiate and make $Q$ bounded. We show that these problems range from quadratic-time to NP-complete, and develop efficient (heuristic) algorithms for them. We also provide an algorithm that, given an effectively bounded SPC query $Q$ and an access schema $\mathcal{A}$, generates a query plan for evaluating $Q$ by accessing a bounded amount of data in any (possibly big) dataset. We experimentally verify that our algorithms substantially reduce the cost of query evaluation.
\end{abstract}

\section{INTRODUCTION}

Query answering is expensive. Consider the problem to decide, given a query $Q$, a dataset $D$ and a tuple $t$, whether $t \in Q(D)$, i.e., whether $t$ is an answer to $Q$ in $D$. This problem is NP-complete for conjunctive queries (i.e., SPC, defined with selection, projection and Cartesian product operators); and it is PSPACE complete for queries in relational algebra $(\mathcal{R} \mathcal{A}$, cf. [6]). When $D$ is big, computing $Q(D)$ is cost-prohibitive. Indeed, even a linear-time query processing algorithm may take days on a dataset $D$ of $\mathrm{PB}$ size $\left(10^{15}\right.$ bytes), and years when $D$ is of EB size ( $10^{18}$ bytes) [21].

This motivates us to ask the following question: is it possible to compute $Q(D)$ by only accessing (visiting and fetching) a small subset $D_{Q}$ of $D$ ? More specifically, we want to

This work is licensed under the Creative Commons AttributionNonCommercial-NoDerivs 3.0 Unported License. To view a copy of this license, visit http://creativecommons.org/licenses/by-nc-nd/3.0/. Obtain permission prior to any use beyond those covered by the license. Contact copyright holder by emailing info@vldb.org. Articles from this volume were invited to present their results at the 40th International Conference on Very Large Data Bases, September 1st - 5th 2014, Hangzhou, China.

Proceedings of the VLDB Endowment, Vol. 7, No. 12

Copyright 2014 VLDB Endowment 2150-8097/14/08. know whether a query $Q$ has the following properties. For all datasets $D$, there exists a subset $D_{Q} \subset D$ such that

(a) $Q\left(D_{Q}\right)=Q(D)$,

(b) $D_{Q}$ consists of no more than $M$ tuples, and

(c) $D_{Q}$ can be effectively identified by using access information, with a cost independent of $|D|$.

Here access information includes indices and cardinality constraints, specified as an access schema $\mathcal{A}$; and $M$ is a bound determined by $\mathcal{A}$ and $Q$ only. We say that $Q$ is effectively bounded under $\mathcal{A}$ if it satisfies all the three conditions above, and bounded if it satisfies conditions (a) and (b) only.

If $Q$ is effectively bounded, then we can find a bounded dataset $D_{Q}$ and compute $Q(D)$ by using $D_{Q}$, independent of the size of possibly big $D$. Moreover, when $D$ grows, the performance does not degrade. In other words, we can reduce big $D$ to a "small" $D_{Q}$ of a manageable size.

Many real-life queries are actually (effectively) bounded.

Example 1: Social networks, e.g., Facebook, allow us to tag a photo and show who is in it. Such a tag is a link to the person "tagged". Consider the following.

(1) A query $Q_{0}$ is to find all photos from an album $a_{0}$ in which a person $u_{0}$ is tagged by one of her friends. The relations needed for answering $Q_{0}$ include the following:

o in_album(photo_id, album_id) for photo albums,

$\circ$ friends(user_id, friend_id) for friends, and

- tagging(photo_id, tagger_id, taggee_id), indicating that taggee_id is tagged by tagger_id in photo_id.

We abbreviate these as in_album( pid $_{1}$, aid), friends(uid, fid) and tagging $\left(\mathrm{pid}_{2}, \mathrm{tid}_{1}, \mathrm{tid}_{2}\right)$, respectively.

Given these, $Q_{0}$ can be written as an SPC query as follows:

$$
\begin{gathered}
Q_{0}\left(\operatorname{pid}_{1}\right)=\pi_{\text {pid }_{1}} \sigma_{C}\left(\text { in_album }_{\left(\operatorname{pid}_{1}, \text { aid }\right) \times \text { friends }(\text { uid }, \text { fid })}\right. \\
\left.\times \operatorname{tagging}\left(\operatorname{pid}_{2}, \operatorname{tid}_{1}, \operatorname{tid}_{2}\right)\right),
\end{gathered}
$$

where the selection condition $C$ is given as

aid $=a_{0} \wedge$ uid $=u_{0} \wedge \operatorname{pid}_{1}=\operatorname{pid}_{2} \wedge \operatorname{tid}_{1}=$ fid $\wedge \operatorname{tid}_{2}=$ uid.

Observe the following. (a) A dataset $D_{0}$ consisting of these relations is possibly big; for instance, Facebook has more than 1 billion users with 140 billion friend links [18]. (b) Query $Q_{0}$ is not bounded: we can add new photos to album $a_{0}$, new friends of $u_{0}$ to friend, or new tuples to tagging, and $Q_{0}$ has to check these tuples when $D_{0}$ grows.

However, social networks often impose limits (cardinality constraints) on $D_{0}$, e.g., (a) each album includes at most 1000 photos, (b) each person may claim up to 5000 friends, and (c) each person in a photo can only be tagged once [19]. 
Moreover, indices can be built on in_album(aid), friends(uid), and tagging $\left(\operatorname{pid}_{1}, \mathrm{tid}_{2}\right)$. As will be seen later, these indices and constraints make an access schema $\mathcal{A}_{0}$.

Under access schema $\mathcal{A}_{0}, Q_{0}$ is effectively bounded: we can compute $Q_{0}\left(D_{0}\right)$ by accessing at most 7000 tuples no matter how large $D_{0}$ is, as follows: (a) select a set $T_{1}$ of at most 1000 pid's from in_album with aid $=a_{0}$, by using the index on in_album(aid); (b) get a set $T_{2}$ of at most 5000 fid's from friends with user_id $=u_{0}$, using the index on friends(uid); (c) using $\operatorname{tid}_{2}=u_{0}$ and pid $_{2}$ 's from $T_{1}$, fetch a set $T_{3}$ of at most $1000\left(\mathrm{pid}_{2}, \mathrm{tid}_{1}\right)$ tuples from tagging via the index on tagging $\left(\operatorname{pid}_{2}, \operatorname{tid}_{2}\right)$; and (d) compute a join $T_{4}$ of $T_{2}$ and $T_{3}$. Then $Q_{0}\left(D_{0}\right)=\pi_{\text {photo_id }}\left(T_{4}\right)$. This query plan visits at most 7000 tuples in total. Moreover, these tuples can be efficiently identified and retrieved by using the indices.

(2) Queries like $Q_{0}$ are routinely posed on social networks. Thus we want a query $Q_{1}$, which is the same as $Q_{0}$ except that uid and aid are not constants, i.e., values $u_{0}$ and $a_{0}$ are not given. Query $Q_{1}$ is not bounded even under $\mathcal{A}_{0}$.

However, $Q_{1}$ can be taken as a parameterized query, a template with parameters (uid, aid, fid, $\mathrm{pid}_{2}, \mathrm{tid}_{1}, \mathrm{tid}_{2}$ ) such that some of them can be substituted with constants when $Q_{1}$ is executed. We identify a minimum subset $X_{P}$ of parameters of $Q_{1}$, referred to as dominating parameters, such that when values of $X_{P}$ are given, $Q_{1}$ is effectively bounded under $\mathcal{A}_{0}$. For instance, uid and aid make a set of dominating parameters: as shown above, when they are instantiated, the query on $D_{0}$ can be answered by accessing at most 7000 tuples. We can find $X_{P}$ and suggest it to users for instantiation.

(3) As another example, consider an arbitrary Boolean SPC query $Q_{2}$ that, given an instance $D$ of a relational schema $\mathcal{R}$, returns true if and only if $Q_{2}(D)$ is nonempty. It is known that $Q_{2}$ is bounded even in the absence of access schema [20]. More specifically, $Q_{2}(D)$ can be computed by accessing at most $\left|Q_{2}\right|$ amount of data no matter how big $D$ is. Indeed, no matter $Q_{2}(D)$ is true or false, it needs a witness $D_{Q}$ of size $|Q|$ such that $Q_{2}\left(D_{Q}\right)=Q_{2}(D)$.

The idea of answering queries with a bounded dataset was first explored in [9-11], and was formalized in [20] (referred to as scale independence there). To make practical use of the idea, several questions have to be settled. Given a query $Q$ and an access schema $\mathcal{A}$, can we determine whether $Q$ is (effectively) bounded under $\mathcal{A}$ ? What is the complexity? If $Q$ is not bounded, can we find a dominating-parameter set $X_{P}$ of $Q$ such that $Q$ becomes effectively bounded under $\mathcal{A}$ when $X_{P}$ is instantiated? Given a dataset $D$, how can we compute $Q(D)$ by efficiently fetching a bounded $D_{Q}$, by using access information in $\mathcal{A}$ ? These questions are nontrivial. It is known that it is undecidable to decide whether $Q$ is bounded for Boolean $\mathcal{R} \mathcal{A}$ queries [20]. The questions are open for SPC queries, which are considered "the most fundamental and the most widely used queries" in practice [23].

Contributions. This paper answers these questions for SPC queries. The main results are as follows.

(1) We formulate bounded SPC queries (Section 2). Following [20], we use an access schema $\mathcal{A}$ to specify indices and cardinality constraints for databases of a relational schema $\mathcal{R}$. We revise the notions of scale independence studied in [20]. We say that an SPC query $Q$ is bounded if for all instances $D$ of $\mathcal{R}$, there exists a $D_{Q} \subset D$ such that
$Q(D)=Q\left(D_{Q}\right)$, and the size of $D_{Q}$ is independent of the size of $D$. If in addition, $D_{Q}$ can be efficiently fetched by using $\mathcal{A}$, then $Q$ is effectively bounded. We show that some queries are bounded but are not effectively bounded.

(2) We study the problems of determining boundedness and effective boundedness (Section 3 ). We provide a set of deduction rules to decide whether an SPC query $Q$ is bounded under an access schema $\mathcal{A}$, and show that the rules provide a sufficient and necessary condition for the boundedness. We also provide a characterization of effectively bounded $Q$ under $\mathcal{A}$. In contrast to $\mathcal{R} \mathcal{A}$ queries [20], these results tell us that there are systematic methods to decide whether SPC queries are bounded or effectively bounded under $\mathcal{A}$.

(3) We study several problems in connection with the (effective) boundedness of SPC queries, establish their complexity, and develop algorithms for them (Section 4). Given an SPC query $Q$ and an access schema $\mathcal{A}$, we study problems to decide (a) whether $Q$ is bounded under $\mathcal{A}$, (b) whether $Q$ is effectively bounded under $\mathcal{A}$, (c) if $Q$ is not effectively bounded, whether there exists a set $X_{P}$ of dominating parameters of $Q$ to make $Q$ effectively bounded under $\mathcal{A}$, and (d) if so, how to find a minimum set $X_{P}$ ? We show that these problems are in $O(|Q|(|\mathcal{A}|+|Q|))$-time, $O(|Q|(|\mathcal{A}|+|Q|))$ time, NP-complete and NPO-complete, respectively. We develop efficient (heuristic) algorithms for these problems.

(4) We give a PTIME (polynomial time) algorithm to generate query plans for answering effectively bounded SPC queries $Q$ under $\mathcal{A}$ (Section 5). The query plans allow us to answer $Q$ in any (possibly big) dataset $D$ by accessing a subset $D_{Q}$ of $D$. The evaluation scales with the size of $D$ : the size $\left|D_{Q}\right|$ of $D_{Q}$ is decided by $\mathcal{A}$ and $Q$ only, and $D_{Q}$ can be fetched by using indices in $\mathcal{A}$ in time independent of $|D|$. We also study the problem for identifying a minimum $D_{Q}$, and show that its decision problem is NP-complete.

(5) We experimentally verify the efficiency and effectiveness of our algorithms, using real-life and synthetic data (Section 6). We find that our algorithms are efficient: they take at most 2.1 seconds to decide whether $Q$ is effectively bounded under $\mathcal{A}$, and to generate a query plan for $Q$, when $Q$ is defined on a relational schema with 19 tables and 113 attributes, and $\mathcal{A}$ consists of 84 constraints. Moreover, our bounded query evaluation approach is effective: on a reallife dataset $D$ of $21.4 \mathrm{~GB}$, our query plan only accesses 3800 tuples and gets answers in 9.3 seconds on average, while MySQL takes longer than 14 hours. That is, our approach is 3 orders of magnitude faster than MySQL. The improvement is more substantial when $D$ grows, since our approach accesses a bounded subset of $D$ no matter how large $D$ is!

These results suggest an approach to answering queries in big data $D$. Given an SPC query $Q$ and an access schema $\mathcal{A}$, we first check in $O(|Q|(|\mathcal{A}|+|Q|))$-time whether $Q$ is effectively bounded under $\mathcal{A}$. If so, we compute $Q(D)$ by accessing a bounded $D_{Q} \subset D$, independent of $|D|$. If not, we may either identify a minimum set of dominating parameters and invite users to supply their values, or suggest users to extend their access schema, such that $Q$ becomes effectively bounded. Only when none of these is possible, we pay the price of computing $Q(D)$ directly in big $D$.

We find that many real-life queries are effectively bounded under a simple access schema, such as (a) parameterized 
queries supported by e-commerce systems, where users issue queries via Web forms by instantiating parameters; and (b) social searches, e.g., the one given in Example 1. Moreover, access schema can be deduced from our familiar functional dependencies (FDs), domain constraints and bounds on reallife data such as those imposed by Facebook (Example 1).

Detailed proofs of the results of the paper are given in [5].

Related work. We characterize related work as follows.

Scale independence. The notion of boundedness is a revision of scale independence proposed in [10], which aims to execute a bounded amount of work in an application regardless of the size of the underlying data. An extension to SQL was proposed in [9] to enforce scale independence, which allows users to specify bounds on the amount of data accessed and the size of intermediate results; when the data required exceeds the bounds, only top- $k$ tuples are retrieved to meet the bounds. Scale independence was also studied in the presence of materialized views [11]. The study differs from our work as follows. (1) Its system [9] is based on key/value store with its own compiler, while we aim to directly improve traditional DBMS. (2) It does not consider effective boundedness and its characterizations. (3) It settles with approximate answers while we focus on exact answers.

The notion of scale independence was recently formalized in [20]. The notion of access schema was also proposed there. For a given bound $M,[20]$ defines a scale independent query $Q$ to be one that for all datasets $D$, there exists $D_{Q} \subseteq D$ such that $Q(D)=Q\left(D_{Q}\right)$ and $\left|D_{Q}\right| \leq M$. It studies several decision problems for scale independence. In particular, it shows that it is undecidable to check whether a Boolean $\mathcal{R} \mathcal{A}$ query is scale independent. It also develops a set of rules as a sufficient condition for deciding whether an $\mathcal{R} \mathcal{A}$ query is scale independent under an access schema.

This works extends [20] as follows. (1) We do not require the size of $D_{Q}$ to be bounded by a predefined $M$. Indeed, if $\left|D_{Q}\right|$ is determined by $\mathcal{A}$ and $Q$ only, its evaluation scales well with $D$. Hence, we define (effectively) bounded queries instead. (2) We provide characterizations for (effectively) bounded SPC queries $Q$ under $\mathcal{A}$, which was not studied in [20]. As opposed to $\mathcal{R} \mathcal{A}$ queries of [20], these give us sufficient and necessary conditions for deciding whether $Q$ is (effectively) bounded. (3) We show that the (effective) boundedness of SPC queries can be decided in PTIME when $M$ is not part of the input, but is NP-complete in the setting of [20] (when $M$ is predefined), in contrast to the undecidability of the problem for $\mathcal{R} \mathcal{A}$ queries. (4) None of the problems for dominating parameters was studied in [20]. (5) We give efficient (heuristic) algorithms for checking whether $Q$ is (effectively) bounded, identifying dominating parameters, and for generating a query plan when $Q$ is effectively bounded. No algorithms were provided in [20].

There has also been work on size bounds for join [12] and conjunctive queries [23]. Given a query $Q$ and a dataset $D$, it is to decide bounds for $|Q(D)|$ in terms of $|Q|$ and $|D|$, possibly in the presence of keys and FDs [23]. Characterizations for deriving worst-case size bounds for these queries are presented there. That line of work differs from ours in both the problems studied and the approaches adopted (e.g., coloring scheme of [23] vs. rule-based inference of ours).

Making big data small. There have been several data reduction schemes that, given a dataset $D$, find a small dataset
$D^{\prime}$ such that one can evaluate queries posed on $D$ by using $D^{\prime}$ instead. These include compression, summarization and data synopses such as histograms, wavelets, quantile summaries, clustering and sampling [7, 14, 17, 22, 24, 25, 27, 29]. Recently BlinkDB [8] has revised the idea to evaluate queries on big data. It adaptively samples data to find approximate query answers within a probabilistic error-bound and time constraints. Similar ideas were also explored in [9].

This work differs from the prior work as follows. (1) We aim to compute exact answers by using a bounded dataset whenever possible, rather than approximate query answers $[8,9]$. (2) The prior reduction schemes $[7,14,17,22,24$, $25,27,29]$ use the same dataset $D^{\prime}$ to answer all queries posed on $D$. In contrast, we adopt a dynamic reduction scheme that finds a small $D_{Q}$ for each query $Q$. Here $D_{Q}$ contains only the information needed for answering $Q$ and hence, allows us to compute $Q(D)$ by using a small dataset $D_{Q}$.

Access schema. Cardinality constraints have been studied for relational data (e.g., [26]). Following [20], this paper aims to identify a bounded dataset $D_{Q}$ to answer a query by making use of available indices and cardinality constraints.

As remarked in [20], access schema is quite different from access patterns $[15,16,28]$. Access patterns require that a relation can only be accessed by providing certain combinations of attribute values. In contrast, access schemas combine indexing and cardinality constraints, and guide us to find a bounded dataset $D_{Q}$ for query answering.

\section{BOUNDED QUERIES UNDER AN AC- CESS SCHEMA}

Below we first review SPC queries, and then present access schemas. Based on these, we define bounded and effectively bounded SPC queries under an access schema.

SPC. Consider a relational schema $\mathcal{R}=\left(R_{1}, \ldots, R_{l}\right)$ in which each $R_{i}$ is a relation schema. Recall that an SPC query over $\mathcal{R}$ has the following form (see, e.g., [6]):

$$
Q(Z)=\pi_{Z} \sigma_{C}\left(S_{1} \times \ldots \times S_{n}\right)
$$

Here $S_{j}$ is a (renaming of a) relation schema in $\mathcal{R}, Z$ is a set of attributes of $\mathcal{R}$, and $C$ is the selection condition of $Q$, defined as a conjunction of equality atoms $x=y$ or $x=c$. where $x, y$ are attributes and $c$ is a constant. We refer to attributes that appear in $Z$ or $C$ as the parameters of $Q$.

To simplify the discussion, we consider $Q$ defined over a single schema $R\left(A_{1}, \ldots, A_{m}\right)$. This does not lose generality due to the lemma below, in which we denote by inst $(\mathcal{R})$ the set of all database instances of relational schema $\mathcal{R}$.

Lemma 1: For any relational schema $\mathcal{R}$, there exist a single relation schema $R$, a linear-time function $g_{D}$ from inst $(\mathcal{R})$ to $\operatorname{inst}(R)$, and a linear-time query-rewriting function $g_{Q}$ from SPC to SPC such that for any instance $D$ of $\mathcal{R}$ and any SPC query $Q$ over $\mathcal{R}, Q(D)=g_{Q}(Q)\left(g_{D}(D)\right)$.

Access schema. An access schema $\mathcal{A}$ over relation schema $R$ is a set of access constraints of the following form:

$$
X \rightarrow(Y, N) \text {, }
$$

where $X$ and $Y$ are sets of attributes of $R$, and $N$ is a natural number. A database $D$ of $R$ satisfies the constraint if

○ for any $X$-value $\bar{a},\left|D_{Y}(X=\bar{a})\right| \leq N$, where $D_{Y}(X=$ $\bar{a})=\{t[Y] \mid t \in D, t[X]=\bar{a}\}$; that is, for each $X$ value, there exist at most $N$ distinct corresponding $Y$ values; 
o there exists an index on $X$ for $Y$ such that given a $X$-value $\bar{a}$, it finds $D^{\prime} \subseteq D$ such that $\left|D^{\prime}\right| \leq N$ and $D_{Y}^{\prime}(X=\bar{a})=D_{Y}(X=\bar{a})$ with a cost measured in $N$.

Here $D^{\prime}$ is one of (possibly many) subsets of $D$ with $N$ tuples, one for each distinct value of $Y$, and $N$ is independent of $|D|$. We say that $D$ satisfies access schema $\mathcal{A}$, denoted by $D \models \mathcal{A}$, if $D$ satisfies all the constraints in $\mathcal{A}$.

An access constraint is a combination of a cardinality constraint and an index. It tells us that for any given $X$-value. there exist a bounded number of corresponding $Y$ values, and the $Y$ values can be efficiently retrieved with the index.

Example 2: Recall from Example 1 the limit of 1000 photos per album. This can be expressed as an access constraint over schema in_album with an index on album_id for photo_id:

$$
\text { album_id } \rightarrow \text { (photo_id, 1000). }
$$

Another constraint over tagging enforces that each person is tagged at most once in a photo: (photo_id, taggee_id) $\rightarrow$ (tager_id, 1). Similarly, the limit of 5000 friends per person is expressed as user_id $\rightarrow$ (friend_id, 5000) over friends.

Observe the following. (a) Functional dependencies (FDs) $X \rightarrow Y$ (see [6]) are a special case of access constraints of the form $X \rightarrow(Y, 1)$ if an index is defined on $X$ for $Y$. (b) Keys are a special form of access constraints $X \rightarrow(R, 1)$, where $R$ denotes all the attributes of relation schema $R$. In general, given an access constraint $X \rightarrow(R, N)$, we can efficiently fetch the entire tuples when an $X$ value is given.

In practice, access constraints can be deduced from the following: (1) FDs; mature techniques are already in place to automatically discover FDs, a special case of access constraints; moreover, the techniques can be extended to discover general access constraints; $(2)$ attributes with bounded domains: if the domain of an attribute $B$ is bounded by $N$ (e.g., each year has 12 months and at most 336 days), then $X \rightarrow(B, N)$ is an access constraint for any set $X$ of attributes; and (3) the semantics of real-life data, e.g., the number of vehicles involved in a road accident is at most 192 from 1979-2005 in the UK (see Section 6 for details).

Bounded and effectively bounded SPC queries. We say that an SPC query $Q$ over relation schema $R$ is bounded under an access schema $\mathcal{A}$ if for all instances $D$ of $R$ that satisfy $\mathcal{A}$, there exists a subset $D_{Q} \subseteq D$ such that

(a) $Q\left(D_{Q}\right)=Q(D)$; and

(b) the size $\left|D_{Q}\right|$ is independent of the size $|D|$ of $D$.

Here $|D|$ is measured as the total number of tuples in $D$.

We say that $Q$ is effectively bounded under $\mathcal{A}$ if $Q$ is bounded under $\mathcal{A}$ and there exists an algorithm that identifies $D_{Q}$ in time determined by $Q$ and $\mathcal{A}$, not by $|D|$.

Intuitively, $Q$ is bounded under $\mathcal{A}$ if it can be answered in a bounded $D_{Q}$. It is effectively bounded if moreover, $D_{Q}$ can be efficiently identified (assuming that given an $X$-value $\bar{a}$, it takes $O(N)$ time to identify $D_{Y}(X=\bar{a})$ in $D$ via an access constraint $X \rightarrow(Y, N)$ in $\mathcal{A})$. For instance, as shown in Example 1, all Boolean SPC queries are bounded even in the absence of access schema, and query $Q_{0}$ is effectively bounded under the access schema $\mathcal{A}_{0}$ of Example 2 .

The result below separates the class $\mathrm{SPC}_{\mathrm{b}}$ of bounded queries from the class $\mathrm{SPC}_{\mathrm{eb}}$ of effectively bounded queries under the same access schema, i.e., $\mathrm{SPC}_{\mathrm{eb}} \subset \mathrm{SPC}_{\mathrm{b}}$.
Proposition 2: There exists a query that is bounded but is not effectively bounded under the same access schema.

\section{CHARACTERIZING EFFECTIVE BOUNDEDNESS}

We now provide sufficient and necessary conditions for determining the (effective) boundedness of SPC queries $Q$ under an access schema $\mathcal{A}$. The main result of the section is as follows. (1) There exists a set $\mathcal{I}_{B}$ of deduction rules such that $Q$ is bounded if and only if it can be proven from $Q$ and $\mathcal{A}$ using $\mathcal{I}_{B}$. (2) Similarly, there exists a set $\mathcal{I}_{E}$ of such rules for effectively boundedness. These yield characterizations of (effective) boundedness via symbolic computation. Moreover, they reveal insight into the boundedness analysis, which helps us develop checking algorithms in Section 4.

We give $\mathcal{I}_{B}$ and $\mathcal{I}_{E}$ in Sections 3.1 and 3.2, respectively.

\subsection{Deduction Rules for Boundedness}

Consider an SPC query $Q(Z)=\pi_{Z} \sigma_{C}\left(S_{1} \times \ldots \times S_{n}\right)$, where $S_{i}$ is a renaming of relation schema $R$. We use $\Sigma_{Q}$ to denote the set of all equality atoms $S[A]=S^{\prime}\left[A^{\prime}\right]$ or $S[A]=c$ derived from the selection condition $C$ of $Q$ by the transitivity of equality. We use $X$ and $X^{\prime}$ to denote sets of attributes of $Q$. We write $\Sigma_{Q} \vdash X=X^{\prime}$ if $X=X^{\prime}$ can be derived from equality atoms in $\Sigma_{Q}$, which can be checked in $O\left(\max \left(|X|,\left|X^{\prime}\right|\right)\right)$ time by leveraging a list of attributes in $Q$ that can be precomputed in $O\left(|Q|^{2}\right)$ time.

To simplify the discussion we assume w.l.o.g. that attributes in $S_{i}$ 's have distinct names via renaming; see, e.g., query $Q_{0}$ of Example 1. We also assume w.l.o.g. that $Q$ is satisfiable, i.e., $\Sigma_{Q}$ does not includes $S[A]=c$ and $S[A]=d$ when $c$ and $d$ are distinct constants.

Rules. We present a set $\mathcal{I}_{B}$ of four deduction rules in Fig. 1. Given an SPC query $Q$ and an access schema $\mathcal{A}$, we write

$$
X \mapsto \mathcal{I}_{B}(Y, N)
$$

if $X \rightarrow(Y, N)$ can be deduced from $\mathcal{A}$ and $\Sigma_{Q}$ by using the rules in $\mathcal{I}_{B}$. Here $X \mapsto \mathcal{I}_{B}(Y, N)$ extends access constraints of Section 2 by allowing $X$ and $Y$ to be sets of attributes of $Q$ from possibly multiple renamed relations of $R$ in $Q$.

One can draw an analogy of $\mathcal{I}_{B}$ to our familiar Armstrong's Axioms for FD implication (see, e.g., [6]).

(1) Reflexivity, Augmentation and Transitivity are immediate extensions of Armstrong's Axioms to access constraints. In particular, Transitivity allows us to propagate boundedness from one relation to another in a Cartesian product $S_{1}\left(X, Y_{1}\right) \times S_{2}\left(Y_{2}, W\right)$ : if for any $X$-value $\bar{a}$, there exist at most $N_{1}$ distinct $Y_{1}$ values, then so do $S_{2}\left[Y_{2}\right]$ by $\Sigma_{Q} \vdash$ $S_{1}\left[Y_{1}\right]=S_{2}\left[Y_{2}\right]$. Then from $Y_{2} \rightarrow\left(W, N_{2}\right)$, it follows that given $\bar{a}$, there exist at most $N_{1} * N_{2}$ distinct $S_{2}[W]$ values.

(2) Actualization is an application of some access constraint of $\mathcal{A}$ to a renaming $S_{i}$ of $R$ that appears in $Q$.

Example 3: Recall relation schemas in_album(pid ${ }_{1}$,aid), friends(uid, fid) and tagging $\left(\operatorname{pid}_{2}, \mathrm{tid}_{1}, \mathrm{tid}_{2}\right)$ given in Example 1. Let $X_{0}$ be (aid, uid, tid 2 , fid, $\operatorname{tid}_{1}$ ).

We show below how $X_{0} \mapsto \mathcal{I}_{B}\left(y, N_{y}\right)$ is proven from query $Q_{0}$ of Example 1 and access schema $\mathcal{A}_{0}$ of Example 2 by using $\mathcal{I}_{B}$, for each parameter $y$ in $Q_{0}$ (i.e., $\sigma_{C}$ or $Z$ ) and for some positive integer $N_{y}$ determined by $Q_{0}$ and $\mathcal{A}_{0}$.
(1) aid $\mapsto \mathcal{I}_{B}\left(\operatorname{pid}_{1}, 1000\right)$
Actualization
(2) $\operatorname{pid}_{2} \mapsto \mathcal{I}_{B}\left(\operatorname{pid}_{2}, 1\right)$
Reflexivity 


\author{
(Reflexivity) If $X^{\prime} \subseteq X$, then $X \mapsto \mapsto_{\mathcal{I}_{B}}\left(X^{\prime}, 1\right)$. \\ (Actualization) If $X \rightarrow(Y, N)$ is in $\mathcal{A}$, then \\ $S_{i}[X] \mapsto \mathcal{I}_{B}\left(S_{i}[Y], N\right)$ for each $i$ in $[1, n]$. \\ (Augmentation) If $X \mapsto \mathcal{I}_{B}(Y, N)$, then \\ $X \cup W \mapsto \mathcal{I}_{B}(Y \cup W, N)$. \\ (Transitivity) If $X \mapsto \mathcal{I}_{B}\left(Y_{1}, N_{1}\right), Y_{2} \mapsto \mathcal{I}_{B}\left(W, N_{2}\right)$, \\ and $\Sigma_{Q} \vdash Y_{1}=Y_{2}$, then $X \mapsto \mathcal{I}_{B}\left(W, N_{1} * N_{2}\right)$.
}

Figure 1: Deduction rules $\mathcal{I}_{B}$ for boundedness

(3) $\Sigma_{Q_{0}} \vdash$ pid1 $=$ pid2

selection condition in $Q_{0}$

(3) aid $\mapsto_{\mathcal{I}_{B}}\left(\right.$ pid $\left._{2}, 1000\right)$

(4) $X_{0} \mapsto \mathcal{I}_{B}$ (aid, 1)

by (1), (2), (3) and Transitivity

Reflexivity

(5) $X_{0} \mapsto \mathcal{I}_{B}\left(\right.$ pid $\left._{2}, 1000\right)$

(3)(2) and Transitivity

Similarly, $X_{0} \mapsto_{\mathcal{I}_{B}}\left(\mathrm{tid}_{1}, 1\right), X_{0} \mapsto \mathfrak{I}_{B}\left(\mathrm{tid}_{2}, 1\right), X_{0} \mapsto \mathcal{I}_{B}$ (uid, 1) and $X_{0} \mapsto \mathcal{I}_{B}$ (fid, 1) by Reflexivity.

Characterization. We next show that $\mathcal{I}_{B}$ provides a sufficient and necessary condition for determining whether an SPC query $Q(Z)$ is bounded under an access schema $\mathcal{A}$.

We use the following notations: (a) $X_{B}$ is the set of all parameters of $Q$ that appear in the selection condition $\sigma_{C}$ such that for any $S[A] \in X_{B}$ and any $z \in Z, \Sigma_{Q} \nvdash S[A]=z$, i.e., attributes that involve in Boolean condition checking but are not part of the output; and (b) $X_{C}$ is the set of all attributes such that for all $S[A] \in X_{C}, \Sigma_{Q} \vdash S[A]=c$ for some constant $c$, i.e., already instantiated with constants.

Theorem 3: An SPC query $Q(Z)$ is bounded under an access schema $\mathcal{A}$ if and only if for each parameter $y$ in $X_{B} \cup Z$, $X_{B} \cup X_{C} \mapsto_{\mathcal{I}_{B}}\left(y, N_{z}\right)$, where $N_{z}$ is a positive integer.

That is, $Q$ is bounded under $\mathcal{A}$ iff for each "free variable" $z \in Z$ of $Q$, its boundedness can be deduced using $\mathcal{I}_{B}$ from (a) those parameters already instantiated in $Q$, and (b) those that only participate in condition checking and hence only need a witness for the truth value of the condition.

Proof: To verify this, we define a notion of access closures. Let $X$ be a set of attributes in $Q$. The access closure $X^{*}$ of $X$ under $\mathcal{A}$ for $Q$ is the set of all attributes $y$ in $Q$ such that for all $D \models \mathcal{A}$, there exists $D^{\prime} \subseteq D$ such that (a) $Q(D)$ $=Q\left(D^{\prime}\right)$, and (b) for all $X$ values $\bar{a},\left|\pi_{y} \sigma_{X=\bar{a}}(D)\right| \leq N_{y}$ for some positive integer $N_{y}$ independent of $|D|$. Here $\sigma_{X=\bar{a}}(D)$ is short for $\sigma_{X_{1}=\bar{a}_{1} \wedge \cdots \wedge X_{n}=\bar{a}_{n}}\left(S_{1} \times \cdots \times S_{n}\right)(D)$, where for each $i \in[1, n]$, (i) $X_{i}$ is the set of attributes in $X$ that are from $S_{i}$; and (ii) $\bar{a}_{i}$ is the set of values in $\bar{a}$ for $X_{i}$.

It suffices to show the following lemmas: under $\mathcal{A}$,

$\circ Q(Z)$ is bounded if and only if $X_{B} \cup Z \subseteq\left(X_{B} \cup X_{C}\right)^{*}$;

$\circ X \mapsto_{\mathcal{I}_{B}}(Y, N)$ for some bound $N$ if and only if $Y \subseteq$ $X^{*}$, for any sets $X$ and $Y$ of attributes in $Q$.

For if these hold, $Q(Z)$ is bounded iff $X_{B} \cup Z \subseteq\left(X_{B} \cup\right.$ $\left.X_{C}\right)^{*}$ iff $X_{B} \cup X_{C} \mapsto_{\mathcal{I}_{B}}\left(X_{B} \cup Z, N\right)$ iff $X_{B} \cup X_{C} \mapsto_{\mathcal{I}_{B}}\left(y, N_{y}\right)$ for each $y \in X_{B} \cup Z$. Hence Theorem 3 follows.

Example 4: For query $Q_{0}(Z)$ given in Example 1, $Z=$ $\left\{\right.$ pid $\left._{1}\right\}, X_{B}=\left\{\operatorname{tid}_{1}\right.$, fid $\}$, and $X_{C}=\{$ uid, aid, tid 2$\}$. By the deduction of $\mathcal{I}_{B}$ given in Example $3, X_{B} \cup X_{C} \mapsto_{\mathcal{I}_{B}}$ ( pid $\left._{1}, 1000\right), X_{B} \cup X_{C} \mapsto \mathcal{I}_{B}\left(\operatorname{tid}_{1}, 1000\right), X_{B} \cup X_{C} \mapsto_{\mathcal{I}_{B}}$ (fid, 1). Hence $Q_{0}$ is bounded under $\mathcal{A}_{0}$ by Theorem 3.

Now consider an arbitrary Boolean SPC query $Q(Z)$ under access schema $\mathcal{A}_{\emptyset}=\emptyset$. The set $Z$ of parameters for projection is $\emptyset$, and $X_{B} \mapsto_{\mathcal{I}_{B}}(x, 1)$ for any $x \in X_{B}$ by Reflexivity. Thus $Q$ is bounded under $\mathcal{A}_{\emptyset}$ by Theorem 3 .
(Reflexivity) If $X^{\prime} \subseteq X$, then $X \mapsto_{\mathcal{I}_{E}}\left(X^{\prime}, 1\right)$.

(Actualization) If $X \rightarrow(Y, N)$ is in $\mathcal{A}$, then $S_{i}[X] \mapsto \mathcal{I}_{E}\left(S_{i}[Y], N\right)$ for each $i$ in $[1, n]$.

(Transitivity) If $X \mapsto_{\mathcal{I}_{E}}(Y, N)$ and $Y \mapsto_{\mathcal{I}_{E}}\left(W, N^{\prime}\right)$, then $X \mapsto_{\mathcal{I}_{E}}\left(W, N * N^{\prime}\right)$.

(Augmentation) If $X \mapsto \mathcal{I}_{E}(Y, N)$ and $X \cup Y$ is indexed, then $X \mapsto_{\mathcal{I}_{E}}(X \cup Y, N)$.

(Combination) If $X_{1} \mapsto_{\mathcal{I}_{E}}\left(Y_{1}, N_{1}\right), \ldots, X_{k} \mapsto_{\mathcal{I}_{E}}\left(Y_{k}, N_{k}\right)$, $\Sigma_{Q} \vdash Y_{1}=Y_{1}^{\prime}, \ldots, \Sigma_{Q} \vdash Y_{k}=Y_{k}^{\prime}$, and $\bigcup_{i=1}^{k}\left(X_{i} \cup Y_{i}^{\prime} \cup Y_{i}\right)$ is indexed in $\mathcal{A}$, then $X_{1} \cup \cdots \cup X_{k} \mapsto_{\mathcal{I}_{E}}\left(Y_{1}^{\prime} \cup \cdots \cup Y_{k}^{\prime}, N_{1} * \cdots * N_{k}\right)$.

Figure 2: Rules $\mathcal{I}_{E}$ for effective boundedness

\subsection{Rules for Effective Boundedness}

To decide whether an SPC query $Q(Z)$ is effectively bounded under $\mathcal{A}$, more needs to be done. When we propagate the boundedness from a set $X$ of attributes to another set $Y$, we have to ensure that the values of $Y$ can be efficiently retrieved via available indices in $\mathcal{A}$. Below we develop a set $\mathcal{I}_{E}$ of deduction rules by incorporating this condition.

Rules. Consider an access schema $\mathcal{A}$ over schema $R$ and a set $Y_{R}$ of attributes of $R$. We say that $Y_{R}$ is indexed in $\mathcal{A}$ if there exists $X_{R} \subseteq Y_{R}$ such that (1) $X_{R} \rightarrow(W, N)$ is an access constraint in $\mathcal{A}$; and (2) $Y_{R} \subseteq X_{R} \cup W$.

If $Y_{R}$ is indexed, given a value $\bar{b}$, we can check whether $Y_{R}$ $=\bar{b}$ is in a dataset $D \models \mathcal{A}$ by using indices in $\mathcal{A}$. Otherwise, we cannot decide this without searching the entire $D$. Thus the condition is necessary for effective boundedness.

Consider an SPC query $Q(Z)=\pi_{Z} \sigma_{C}\left(S_{1} \times \ldots \times S_{n}\right)$ and a set $Y=\left(Y_{1}, \ldots, Y_{n}\right)$ of parameters in $Q$ (i.e., in $C$ or $\left.Z\right)$, where $Y_{i}$ consists of attributes from $S_{i}$. We say that $Y$ is indexed in $\mathcal{A}$ if each $Y_{i}$ is indexed in $\mathcal{A}$.

Using these, we give a set $\mathcal{I}_{E}$ of five rules for deducing the effective boundedness of SPC queries, in Fig. 2. We define $X \mapsto \mathcal{I}_{E}(Y, N)$ along the same lines as $X \mapsto_{\mathcal{I}_{B}}(Y, N)$, using $\mathcal{I}_{E}$. While Reflexivity, Actualization and Transitivity of $\mathcal{I}_{E}$ are the same as their counterparts in $\mathcal{I}_{B}$, the others are not.

(1) Augmentation in $\mathcal{I}_{E}$ revises its counterpart in $\mathcal{I}_{B}$ by allowing $Y$ to be extended with only indexed attributes.

(2) Combination also restricts Augmentation of $\mathcal{I}_{B}$ by enforcing the indexing condition; i.e., for any $X_{i}$-value $\bar{a}_{i}$, if $\bar{a}_{i}$ is in $\pi_{X_{i}}(D)$ for a dataset $D \models \mathcal{A}$, then the deduced $Y$ value must be in $\pi_{Y}(D)$ and can be retrieved via indices. Note that Augmentation is a special case of Combination; we opt to keep Augmentation in $\mathcal{I}_{E}$ as it is easier to use.

Characterization. Based on $\mathcal{I}_{E}$, we give a sufficient and necessary condition for effective boundedness. For an SPC query $Q(Z)=\pi_{Z} \sigma_{C}\left(S_{1} \times \ldots \times S_{n}\right)$, we use the following notations: for all $i \in[1, n]$, (a) $X_{C}^{i}$ is the set of all attributes of $S_{i}$ already instantiated in $Q$, i.e., $X_{C}^{i}=$ $\left\{S_{i}[A] \in S_{i} \mid \Sigma_{Q} \vdash S_{i}[A]=c\right.$ for a constant $\left.c\right\}$, where $S_{i}$ denotes the set of all attributes of $S_{i}$; (b) $X_{C}=X_{C}^{1} \cup \cdots \cup X_{C}^{n}$; (c) $X_{Q}^{i}$ denotes the set of all parameters of $S_{i}$ that appear in either $C$ or $Z$ of $Q$; and (d) $\mathcal{X}^{\mathcal{A}}$ is the set of subsets $S_{i}[X]$ of attributes such that $X \rightarrow(Y, N)$ is in $\mathcal{A}$, for all $i \in[1, n]$.

Theorem 4: An SPC query $Q(Z)$ is effectively bounded under an access schema $\mathcal{A}$ if and only if for each $i \in[1, n]$,

(1) $X_{C}^{i} \subseteq W$ for some $W \in \mathcal{X}^{\mathcal{A}}$; and 
(2) $X_{C} \mapsto \mathcal{I}_{E}\left(X_{Q}^{i}, N_{i}\right)$ for some natural number $N_{i}$ that is determined by $Q$ and $\mathcal{A}$ only.

That is, the instantiated attributes $X_{C}^{i}$ can be checked using indices, as well as those attributes that participate in output or Boolean conditions of $Q$. We will use this characterization to generate query plans in Section 5 . We show the result by using a notion of effective access closures [5].

Example 5: We show that $Q_{0}\left(\right.$ pid $\left._{1}\right)$ of Example 1 is effectively bounded under access schema $\mathcal{A}_{0}$ of Example 2. First,

(1) aid $\mapsto_{\mathcal{I}_{E}}\left(\operatorname{pid}_{1}, 1000\right)$

(2) aid $\mapsto \mathcal{I}_{E}\left(\left(\right.\right.$ aid, pid $\left.\left._{1}\right), 1000\right)$

(3) (aid, uid) $\mapsto_{\mathcal{I}_{E}}$ (aid, 1)

(4) (aid, uid) $\mapsto_{\mathcal{I}_{E}}\left(\left(\right.\right.$ aid, pid $\left.\left._{1}\right), 1000\right)$

(5) uid $\mapsto_{\mathcal{I}_{E}}$ (fid, 5000)

(6) uid $\mapsto \mathcal{I}_{E}$ ((uid, fid), 5000)

(7) (aid, uid) $\mapsto_{\mathcal{I}_{E}}$ (uid, 1)

(8) (aid, uid) $\mapsto_{\mathcal{I}_{E}}$ ((uid, fid), 5000)

(9) uid $\mapsto \mathcal{I}_{E}$ (uid, 1)

(10) (aid, uid) $\mapsto_{\mathcal{I}_{E}}\left(\left(\right.\right.$ pid $\left.\left._{2}, \mathrm{tid}_{2}\right), 1000\right)$

(11) $\left(\operatorname{pid}_{2}, \operatorname{tid}_{2}\right) \mapsto \mathcal{I}_{E}\left(\operatorname{tid}_{1}, 1\right)$

Actualization

(1) and Augmentation Reflexivity

(3)(2) and Transitivity

Actualization

Augmentation

Reflexivity

(7)(6) and Transitivity

Reflexivity

(1)(9) and Combination

Actualization

(12) $\left(\operatorname{pid}_{2}, \mathrm{tid}_{2}\right) \mapsto_{\mathcal{I}_{E}}\left(\left(\mathrm{pid}_{2}, \mathrm{tid}_{1}, \mathrm{tid}_{2}\right), 1\right)$ Augmentation

$\underline{(13) \text { (aid, uid }) \mapsto \mathcal{I}_{E}\left(\left(\text { pid }_{2}, \text { tid }_{1}, \text { tid }_{2}\right),\right.}, \quad(10)(12)$ and Transitivity $\underline{1000})$

Then (a) condition (1) of Theorem 4 is satisfied since aid, uid and $\mathrm{tid}_{2}$ are in subsets $\{$ aid $\},\{$ udi $\}$ and $\left\{\right.$ pid $\left._{2}, \operatorname{tid}_{2}\right\}$ of $\mathcal{X}^{\mathcal{A}_{0}}$, respectively. (b) Condition (2) is satisfied by deduction steps (4), (8) and (13) above, and as $\left(\mathrm{pid}_{2}, \mathrm{tid}_{2}\right)$ is indexed in $\mathcal{A}_{0}$. Thus $Q_{0}$ is effectively bounded under $\mathcal{A}_{0}$ by Theorem 4 .

Remark. Note that we do not need "full and complete" access schema to achieve "boundedness". Instead, as will be shown in Section 6, in practice many (parameterized) queries on real-life data are effectively bounded, under only a small number of access constraints.

\section{BOUNDEDNESS: COMPLEXITY AND ALGORITHMS}

We next study two issues in connection with the (effective) boundedness of SPC queries. (1) We study the complexity and algorithms for deciding whether an SPC query is (effectively) bounded under an access schema $\mathcal{A}$. (2) When $Q$ is not effectively bounded, we study whether $Q$ can be made effectively bounded under $\mathcal{A}$ by instantiating a set $X_{P}$ of parameters of $Q$, and if so, how to compute a minimum $X_{P}$.

The main results of this section are as follows. (1) The boundedness of $Q$ under $\mathcal{A}$ can be decided in quadratic time (Section 4.1). (2) The same complexity holds for effective boundedness (Section 4.2). (3) The decision problem for dominating parameters is NP-complete, and its optimization problem is NPO-complete. We provide an efficient heuristic algorithm to compute dominating parameters (Section 4.3).

\subsection{Checking Boundedness}

We start with the boundedness problem $\operatorname{Bnd}(Q, \mathcal{A})$ :

- Input: A relation schema $R$, an SPC query $Q$ over $R$, and an access schema $\mathcal{A}$ over $R$.

- Question: Is $Q$ bounded under $\mathcal{A}$ ?

This is to decide whether for all datasets $D$ that satisfy $\mathcal{A}$, there exists at all a subset $D_{Q}$ such that $Q(D)=Q\left(D_{Q}\right)$ and $\left|D_{Q}\right|$ is independent of the size $|D|$ of the underlying $D$.

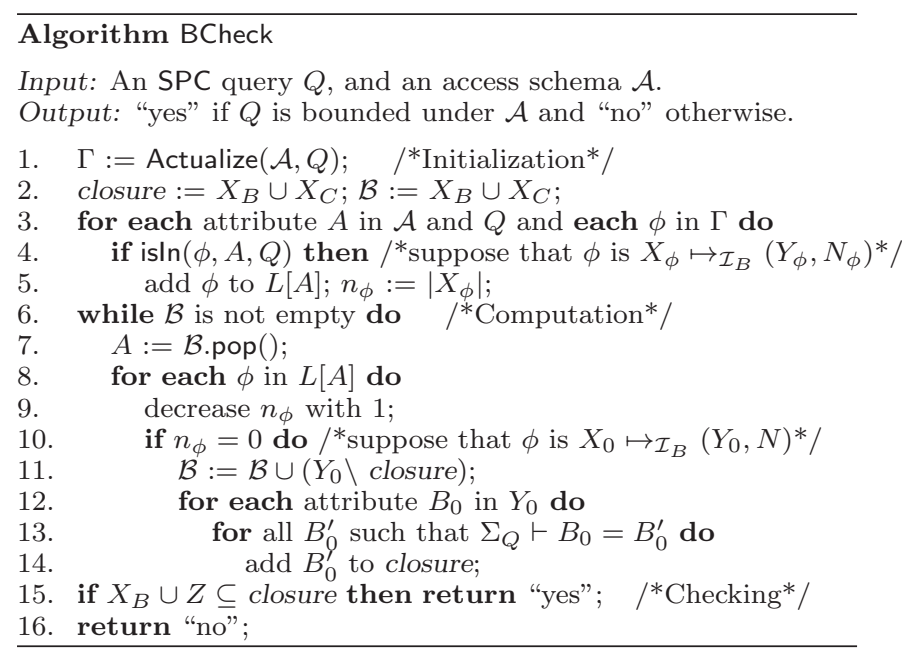

Figure 3: Algorithm BCheck

While this problem is undecidable for (Boolean) $\mathcal{R} \mathcal{A}$ queries [20], it is decidable in PTIME for SPC.

Theorem 5: For any SPC query $Q$ and access schema $\mathcal{A}$, $\operatorname{Bnd}(Q, \mathcal{A})$ can be decided in $O(|Q|(|\mathcal{A}|+|Q|))$ time.

Here $|\mathcal{A}|$ and $|Q|$ are the size of $\mathcal{A}$ and $Q$, respectively, and are typically small in practice, compared to datasets $D$.

As a constructive proof for Theorem 5 , we next give such an algorithm for checking the boundedness of $Q$ under $\mathcal{A}$.

Algorithm BCheck. The algorithm is denoted by BCheck and shown in Fig. 3. It is based on the characterization of $\mathcal{I}_{B}$ (Section 3 ). It computes $\left(X_{B} \cup X_{C}\right)^{*}$, stored in a variable closure, and concludes that $Q$ is bounded under $\mathcal{A}$ if and only if $X_{B} \cup Z \subseteq$ closure, i.e., when all parameters of $Q$ are covered by $\left(X_{B} \cup X_{C}\right)^{*}$ (see Theorem 3 and its proof).

More specifically, BCheck first actualizes access constraints of $\mathcal{A}$ in each renaming $S_{i}$ of schema $R$ in $Q$ : for each $X \rightarrow(Y, N)$ in $\mathcal{A}$ and each $S_{i}$ in $Q$, it includes $S_{i}[X] \mapsto_{\mathcal{I}_{B}}$ $\left(S_{i}[Y], N\right)$ in a set $\Gamma$ (line 1$)$. Using $\Gamma$, it then computes closure (lines 2-14) such that if $X_{B} \cup X_{C} \mapsto \mathcal{I}_{B}(y, N)$ for some $N$ and attribute $y$, then $y$ is included in closure. After this, it simply checks whether $X_{B} \cup Z$ is contained in closure; it returns "yes" if so and "no" otherwise (lines 15-16).

We next show how BCheck computes closure, starting with auxiliary structures used by BCheck.

Auxiliary structures. BCheck uses three auxiliary structures.

(1) BCheck maintains a set $\mathcal{B}$ of attributes in $\mathcal{A}$ and $Q$ that are in closure but it remains to be checked what other attributes can be deduced from them via $\mathcal{I}_{B}$. Initially, $\mathcal{B}=$ $X_{B} \cup X_{C}$ (line 2). BCheck uses $\mathcal{B}$ to control the while loop (lines 6-14): it terminates when $\mathcal{B}=\emptyset$, i.e., when all necessary deduction checking via $\mathcal{I}_{B}$ has been completed.

(2) For each constraint $\phi: X \mapsto \mathcal{I}_{B}(Y, N)$ in $\Gamma$, BCheck maintains a counter $n_{\phi}$ to keep track of those attributes of $X$ that are still in $\mathcal{B}$. Initially, $n_{\phi}$ is the number of attributes in $X$. When $n_{\phi}=0$, i.e., after all $X$ attributes have been processed, the $Y$ attributes can be added to $\mathcal{B}$ (lines 10-11).

(3) For each attribute $A$ in $Q$ and $\Gamma$, BCheck uses a list $L[A]$ to store all constraints $X \mapsto \mathcal{I}_{B}(Y, N)$ in $\Gamma$ such that either $A$ is in $X$ or there exists $A^{\prime}$ in $X$ with $\Sigma_{Q} \vdash A=A^{\prime}$. That is, $L[A]$ indexes constraints that are "applicable" to $A$. 
Computing closure. With these structures, BCheck computes closure as follows. It first initializes the auxiliary structures as described above (lines 2-5). Here function is $\ln (\phi, A, Q)$ checks whether constraint $\phi: X_{\phi} \mapsto \mathcal{I}_{B}\left(Y_{\phi}, N_{\phi}\right)$ is "applicable" to attribute $A$, i.e., whether there exists $A^{\prime}$ such that $\Sigma_{Q} \vdash A=A^{\prime}$ and $A^{\prime}$ is in $X_{\phi}$ (line 5).

After this, BCheck processes attributes in $\mathcal{B}$ one by one (lines 6-14). For each attribute $A \in \mathcal{B}$ and each constraint $\phi: X_{0} \mapsto_{\mathcal{I}_{B}}\left(Y_{0}, N\right)$ in $L[A]$, it decreases the counter $n_{\phi}$ by 1 . When $n_{\phi}=0$, i.e., all attributes in $X_{0}$ have been inspected, BCheck conducts deduction via $\mathcal{I}_{B}$ (lines 11-14). It adds to $\mathcal{B}$ attributes $B_{0}$ in $Y_{0}$ that are not yet in closure (line 11), and add to closure all those attributes $B_{0}^{\prime}$ such that $\Sigma_{Q} \vdash B_{0}=B_{0}^{\prime}$ (lines 12-14). When $\mathcal{B}$ becomes empty, BCheck returns "yes" iff $X_{B} \cup Z \subseteq$ closure (lines 15-16).

Correctness 8 Complexity. The correctness of BCheck follows from Theorem 3. To see that BCheck is in $O(|Q|(|A|+$ $|Q|))$ time, observe the following. (1) The initialization steps take $O(|Q \| \mathcal{A}|)$ time (lines 1-5). (2) The closure is computed in $O(|\mathcal{A}|+|\mathcal{A}||Q|)$ time (lines 6-14), since the counters are updated at most $O(|\mathcal{A}||Q|)$ times in total, and each $\phi$ in $\Gamma$ is used at most once, in $O(|\phi|+|Q|)$ time (thus $O(|\mathcal{A}|+|\mathcal{A}||Q|$ ) time in total). (3) The checking (line 15) can be done in $O\left(|Q|^{2}\right)$ time, since the size of closure is bounded by $O(|Q|)$.

Example 6: We show how algorithm BCheck finds that query $Q_{0}$ of Example 1 is bounded under the access schema $\mathcal{A}_{0}$ of Example 2. Here $X_{B} \cup X_{C}=$ \{aid, uid, tid $_{2}$, fid, tid $\left._{1}\right\}$. BCheck initializes $\Gamma$ with aid $\mapsto_{\mathcal{I}_{B}}\left(\operatorname{pid}_{1}, 1000\right)\left(\phi_{1}\right)$, $\left(\operatorname{pid}_{2}, \operatorname{tid}_{2}\right) \mapsto_{\mathcal{I}_{B}}\left(\operatorname{tid}_{1}, 1\right)\left(\phi_{2}\right)$, and uid $\mapsto_{\mathcal{I}_{B}}\left(\right.$ fid, 5000) $\left(\phi_{3}\right)$. It assigns $X_{B} \cup X_{C}$ as the initial value of closure and $\mathcal{B}$, and sets counters $n_{\phi_{1}}=n_{\phi_{3}}=1, n_{\phi_{2}}=2$. After aid is popped off from $\mathcal{B}, n_{\phi_{1}}$ is decreased to 0 and BCheck updates closure and $\mathcal{B}$ with $\phi_{1}$ (lines 11-14). Since $\Sigma_{Q} \vdash \operatorname{pid}_{1}=\operatorname{pid}_{2}$, both $\operatorname{pid}_{1}$ and $\operatorname{pid}_{2}$ are added to closure, and pid $_{1}$ is added to $\mathcal{B}$. After this iteration, closure remains unchanged and $\mathcal{B}$ will be reduced to empty. Since $X_{B} \cup Z=\left\{\right.$ pid $_{1}, \operatorname{pid}_{2}, \operatorname{tid}_{1}$, fid $\}$ is a subset of closure, BCheck returns "yes".

\subsection{Checking Effective Boundedness}

We next study the effective boundedness problem, denoted by $\operatorname{EBnd}(Q, \mathcal{A})$ and stated as follows:

- Input: $R, Q$ and $\mathcal{A}$ as in $\operatorname{Bnd}(Q, \mathcal{A})$.

○ Question: Is $Q$ effectively bounded under $\mathcal{A}$ ?

It is to decide whether for any $D$ that satisfies $\mathcal{A}$, we can fetch $D_{Q} \subseteq D$ via indices in $\mathcal{A}$ such that $Q(D)=Q\left(D_{Q}\right)$.

Problem EBnd is also decidable in quadratic-time.

Theorem 6: $\operatorname{EBnd}(Q, \mathcal{A})$ is in $O(|Q|(|\mathcal{A}|+|Q|))$ time.

We prove Theorem 6 by providing an algorithm for checking the effective boundedness of $Q$ under $\mathcal{A}$.

Algorithm EBCheck. The algorithm, denoted by EBCheck, extends algorithm BCheck by leveraging Theorem 4 and the following connection between $\mathcal{I}_{E}$ and the access closure for boundedness: for any sets $X$ and $Y$ of attributes in $Q$ such that $X \subseteq Y, X \mapsto \mathcal{I}_{E} Y$ if and only if $Y \subseteq X^{*}$ and $Y$ is indexed in $\mathcal{A}$. Based on this, EBCheck works as follows.

Step 1 (computing closure): Compute $X_{C}^{*}$ by adopting the closure computation part of BCheck (lines 1-14, Fig. 3) except that it initializes closure to be $X_{C}$ instead of $X_{B} \cup X_{C}$.

Step 2 (checking): Check (a) whether $\bigcup_{i=1}^{n} X_{Q}^{i}$ is a subset of $X_{C}^{*}$ and (b) whether $\bigcup_{i=1}^{n} X_{Q}^{i}$ is indexed in $\mathcal{A}$. If so, $Q$ is effectively bounded under $\mathcal{A}$. Note that the condition (1) of Theorem 4 is implied by (b) here.

As both steps are in $O(|Q|(|\mathcal{A}|+|Q|)$ time, so is EBCheck.

Example 7: Consider again query $Q_{0}$ of Example 1 and access schema $\mathcal{A}_{0}$ of Example 2. The deduction analysis of Example 5 tells us that $X_{C}^{*}$ of $Q_{0}$ covers parameters of in_album, friends and tagging; moreover, $X_{C}^{*}$ is indexed by $\mathcal{A}_{0}$. That is, the conditions in Step 2 of EBCheck are satisfied. Hence, $Q_{0}$ is effectively bounded under $\mathcal{A}_{0}$.

\subsection{Computing Dominating Parameters}

As illustrated in Example 1, when an SPC query $Q$ is not effectively bounded under $\mathcal{A}$, we want to identify a minimum set $X_{P}$ of parameters of $Q$ such that if $X_{P}$ is instantiated, $Q$ becomes effectively bounded. We want to find and suggest such an $X_{P}$ to users if it exists. When the users provide a value of $X_{P}, Q$ can be answered in a big dataset $D$ by accessing a bounded amount of data. We consider parameters of $X_{P}$ that are not in $X_{C}$, i.e., not yet instantiated in $Q$, and are not trivial, i.e., not covering all attributes in $Q$.

More specifically, we use $Q\left(X_{P}=\bar{a}\right)$ to denote the query obtained from $Q$ when $X_{P}$ is given a value $\bar{a}$. We call $X_{P}$ a set of dominating parameters of $Q$ under $\mathcal{A}$ w.r.t.any fixed fraction $\alpha \in(0,1)$, if $\left|X_{P}\right| /\left|X_{B}\right| \leq \alpha$ and $Q\left(X_{P}=\bar{a}\right)$ is effectively bounded under $\mathcal{A}$ for all given $X_{P}$ values $\bar{a}$ (see Section 3.1 for $X_{B}$ ). Intuitively, by instantiating $X_{P}$, which contains at most $\alpha\left|X_{B}\right|$ attributes of $Q$, we can make $Q\left(X_{P}=\bar{a}\right)$ effectively bounded under $\mathcal{A}$.

Problems and complexity. This suggests that we study the following decision and optimization problems.

The dominating parameter problem $\operatorname{DP}(Q, \mathcal{A})$.

- Input: $R, Q(Z), \mathcal{A}$ as in $\operatorname{EBnd}(Q, \mathcal{A})$, any fixed $\alpha$.

- Question: Does there exist a set of dominating parameters of $Q$ under $\mathcal{A}$ w.r.t. $\alpha$ ?

The minimum dominating parameter problem $\operatorname{MDP}(Q, \mathcal{A})$.

- Input: $R, Q(Z), \mathcal{A}$ as in $\operatorname{EBnd}(Q, \mathcal{A})$, any fixed $\alpha$.

- Output: A set of dominating parameters $X_{P}$ of $Q$ un$\operatorname{der} \mathcal{A}$ w.r.t. $\alpha$ with minimum cardinality, if it exists.

Problem $\operatorname{DP}(Q, \mathcal{A})$ is to decide whether $Q$ has a set of dominating parameters at all. $\operatorname{Problem} \operatorname{MDP}(Q, \mathcal{A})$ is to compute a minimum set of dominating parameters of $Q$.

Example 8: An SPC query may not have a set of dominating parameters under an access schema. As an example, consider query $Q_{0}$ of Example 1 and an access schema $\mathcal{A}_{1}$ that contains all access constraints in $A_{0}$ of Example 2 except (photo_id, taggee_id) $\rightarrow$ (tagger_id, 1$)$. Then $Q_{0}$ is not effectively bounded under $\mathcal{A}_{1}$, and worse still, no matter what parameters of $Q_{0}$ we instantiate, it is still not effectively bounded. This is because no index is built on tagging in $\mathcal{A}_{1}$, and hence we cannot verify, e.g., whether $\operatorname{tid}_{2}=u_{0}$ is in a tagging instance without searching the entire $D$.

While DP and MDP are important, they are hard.

Theorem 7: For SPC query $Q$ and access schema $\mathcal{A}$,

(1) $\mathrm{DP}(Q, \mathcal{A})$ is NP-complete; and

(2) $\operatorname{MDP}(Q, \mathcal{A})$ is $\mathrm{NPO}$-complete.

NPO is the class of all NP optimization problems. NPOcomplete problems are the hardest optimization problems 
in NPO: they do not even allow PTIME approximation algorithms with an exponential approximation ratio (cf. [13]).

Algorithm. In light of Theorem 7, we develop a heuristic algorithm that for any fixed $\alpha \in(0,1)$, given $Q$ and $\mathcal{A}$, checks whether there exists a set of dominating parameters for $Q$ under $\mathcal{A}$ w.r.t. $\alpha$; it finds and returns such a set $X_{P}$ if so, and returns "no" otherwise. The algorithm, denoted by findDP $\mathrm{h}_{\mathrm{h}}$, consists of three steps.

Step 1 (initial candidates): For each renaming $S_{i}$ of $R$ in $Q$ and each parameter $A$ of $Q$ that is in $S_{i}$ but is not in $X_{C}$, add $A$ to a set $X_{P}$ if there exists a constraint $X \rightarrow(Y, N)$ in $\mathcal{A}$ such that $A$ is in $S_{i}[X] \cup S_{i}[Y]$.

Step 2 (checking): Check (a) whether $\bigcup_{i=1}^{n} X_{Q}^{i}$ is indexed in $\mathcal{A}$ and (b) whether for all $X_{Q}^{i}, X_{Q}^{i} \subseteq X_{P}$ (see Section 3.2 for the definition of $X_{Q}^{i}$ ). If not, return "no".

Step 3 (minimizing): We optimize $X_{P}$ iteratively as follows. Each time we pick one attribute $A$ of some $S_{i}$ from $X_{P}$, and check whether there is $X \rightarrow(Y, N)$ in $\mathcal{A}$ such that $S_{i}[X] \subseteq$ $X_{P}, A \notin S_{i}[X]$ and $A \in S_{i}[Y]$. If so, let $X_{P}=X_{P} \backslash \operatorname{ext}_{Q}(A)$ since $X_{P}$ can be recovered from $X_{P} \backslash\{A\}$ via deduction of $\mathcal{I}_{E}$, where $\operatorname{ext}_{\mathrm{Q}}(A)$ consists of all parameters $x$ such that $\Sigma_{Q} \vdash A=x$. We then process the next attribute. We return $X_{P}$ when it cannot be further reduced and $\left|X_{P}\right| /\left|X_{B}\right| \leq \alpha$.

Correctness \& Complexity. One can verify that if findDP $\mathrm{P}_{\mathrm{h}}$ returns $X_{P}$, then $X_{P}$ is a set of dominating parameters for $Q$ under $\mathcal{A}$. Indeed, if $X_{P}$ is instantiated, then for all $S_{i}$ in $Q$, all parameters in $X_{Q}^{i}$ can be deduced from $X_{P}$ via $\mathcal{I}_{E}$ and are also indexed. Hence $Q\left(X_{P}=\bar{a}\right)$ is effectively bounded under $\mathcal{A}$ by Theorem 4 , for any $X_{P}$ value $\bar{a}$.

Algorithm findDP $\mathrm{h}$ is in $O(|Q|(|Q|+|\mathcal{A}|))$ time. Indeed, its step 1 is in $O(|\mathcal{A}||Q|)$ time; step 2 takes $O\left(|Q|^{2}\right)$ time since $\left|X_{P}\right|$ and $\left|X_{Q}\right|$ are both bounded by $|Q|$; and step 3 is in $O(|Q|(|\mathcal{A}|+|Q|))$ time because $\left|X_{P}\right| \leq|Q|$; hence it takes $O(|\mathcal{A}|)$ time to check whether an attribute $A$ can be removed from $X_{P}$, and $O(|Q|)$ time to remove $\operatorname{ext}_{\mathrm{Q}}(A)$.

Example 9: Recall that query $Q_{1}$ of Example 1 is not effectively bounded under access schema $\mathcal{A}_{0}$ of Example 2. Consider $\alpha=3 / 7$. We show how findDP $\mathrm{P}_{\mathrm{h}}$ finds a set $X_{P}$ of dominating parameters for $Q_{1}$. In step 1 , it sets $X_{P}$ $=\left\{\operatorname{pid}_{1}\right.$, aid, uid, fid, $\left.\operatorname{pid}_{2}, \operatorname{tid}_{1}, \mathrm{tid}_{2}\right\}$. In step 2 , findDP $\mathrm{h}$ finds $X_{Q}^{i}$ contained in $X_{P}$ for $X_{Q}^{i}$ in in_album, friends and tagging; hence there exists a set of dominating parameters for $Q_{1}$. In step 3 , it reduces $X_{P}$. (a) It first finds that album_id $\rightarrow$ (photo_id, 1000) in $\mathcal{A}_{0}$, and removes pid ${ }_{1}$ and pid $_{2}$ from $X_{P}$ since $\Sigma_{Q} \vdash$ pid $_{1}=$ pid $_{2}$. (b) It then finds user_id $\rightarrow$ (friend_id, 5000) in $\mathcal{A}_{0}$, and removes fid and tid ${ }_{1}$ from $X_{P}$ by $\Sigma_{Q} \vdash$ fid $=$ tid $_{1}$. Finally, findDP $\mathrm{h}_{\mathrm{h}}$ finds that it can remove no more parameters from $X_{P}$ and $\left|X_{P}\right| /\left|X_{B}\right| \leq \alpha$, and thus returns $X_{P}=\left\{\right.$ aid, uid, $\left.\operatorname{tid}_{2}\right\}$, which is exactly the set of instantiated parameters for $Q_{0}$ (by $\Sigma_{Q_{0}} \vdash \mathrm{tid}_{2}=\mathrm{u}_{0}$ ).

\section{ALGORITHM FOR EFFECTIVELY BOUNDED QUERIES}

Algorithm EBCheck of Section 4.2 is able to determine the effective boundedness of SPC queries. However, it does not tell us how to identify a bounded amount of data to answer those queries. To bridge the gap, we next develop an algorithm that, given an effectively bounded SPC query $Q(Z)=\pi_{Z} \sigma_{C}\left(S_{1} \times \ldots \times S_{n}\right)$ and an access schema $\mathcal{A}$, finds a query plan that, given a (big) dataset $D$, fetches a bounded
$D_{Q} \subseteq D$ using indices in $\mathcal{A}$ such that $Q(D)=Q\left(D_{Q}\right)$.

The main results of the section are as follows. (1) There exists an $O\left(|Q|^{2}|\mathcal{A}|^{3}\right)$-time algorithm that generates query plans for effectively bounded SPC queries (Section 5.1). (2) We also study the problem to find a minimum bounded $D_{Q}$, and show that the problem is NP-complete (Section 5.2).

\subsection{Determining and Computing $D_{Q}$}

We find a query plan for $Q$ by deducing a proof $\rho_{i}$ for $X_{C} \mapsto \mathcal{I}_{E}\left(X_{Q}^{i}, M_{i}\right)$ for all $i \in[1, n]$, following Theorem 4 . Below we show that the proofs yield a query plan that, for any dataset $D$ such that $D \models \mathcal{A}$, tells us how to find $D_{Q}$ such that $Q(D)=Q\left(D_{Q}\right)$ and $D_{Q}$ has at most $\sum_{i=1}^{n} M_{i}$ tuples.

Query plan from proofs. Suppose that $X_{C} \mapsto \mathcal{I}_{E}\left(X_{Q}^{i}, M_{i}\right)$ is proven by $\rho_{i}=\varphi_{1}, \cdots, \varphi_{m}$, where $\varphi_{j}$ denotes application of a rule in $\mathcal{I}_{E}$. We show that given $D, \rho_{i}$ tells us how to find a list of subsets $T_{1}, \ldots, T_{m}$ of $D$ such that

○ $D_{Q}^{i}=\bigcup_{j=1}^{m} T_{j}$ and $D_{Q}=\bigcup_{i=1}^{n} D_{Q}^{i}$, and

○ for all $j \in[1, m], T_{j} \subseteq D, T_{j}$ has at most $N_{j}$ tuples and can be fetched by using indices in $\mathcal{A}$, where $N_{j}$ is a number deduced from the proof, independent of $|D|$.

We can then compute $Q(D)$ by conducing joins and projections on these $T_{j}$ 's only, guided by conditions in $\sigma_{C}$ of $Q$, as illustrated by how we get $Q_{0}\left(D_{0}\right)$ using $T_{1}-T_{4}$ in Example 1 .

Below we show how to fetch $T_{j}$ from $D$ guided by rule $\varphi_{j}$, by giving two example rules (see [5] for other rules). Initially, $T_{1}=\bigcup_{j=1}^{n} \sigma_{X_{j}=C_{j}}(D)$, and can be fetched by using indices in $\mathcal{A}$ on the constants of $X_{C}$ (see Theorem 4 and its proof).

(a) When $\varphi_{j}$ actualizes a constraint $X \rightarrow(Y, N)$ of $\mathcal{A}$, we fetch $N$ tuples for $T_{j}$ either from $D$ by using index in $\mathcal{A}$ on $X$ for $Y$, or from a bounded subset $T_{j^{\prime}}$ of $D\left(j^{\prime}<j\right)$ deduced from previous steps in proof $\rho_{i}$, on which $\varphi_{j}$ is applied.

(b) When $\varphi_{j}$ is Combination, we get $T_{j}$ as follows. Denote $\cup_{s=1}^{j-1} T_{s}$ by $T$. As indicated by the rule (Fig. 2), for $l \in[1, k]$, (i) all $X_{l}$ and $Y_{l}$ values are already fetched in $T$; and (ii) we can check whether these $X_{l}$ and $Y_{l}$ values appear in tuples of $D$, i.e., they are contained in the projection of $D$ on $\bigcup_{l=1}^{k} X_{l} \cup Y_{l}^{\prime}$, by using the indices on the attributes. There are at most $N_{1} * \ldots * N_{k}$ such tuples from $T$ to be inspected in $D$, and $T_{j}$ consists of these tuples.

Algorithm QPlan. We now present the algorithm, denoted by QPlan and shown in Fig. 4. Based on the connection between $\mathcal{I}_{E}$ proofs and query plans given above, QPlan focuses on finding a proof $\rho_{i}$ for each $X_{C} \mapsto_{\mathcal{I}_{E}}\left(X_{Q}^{i}, M_{i}\right)$, based on the characterization of $\mathcal{I}_{E}$ of Section 3. It represents $\rho_{i}$ as an object $o_{i}$, which consists of three components:

○ $o_{i} . X$ : parameters of $X_{Q}^{i}$ deduced from the proof;

○ $o_{i} \cdot \mathcal{P}$ : a proof for deducing $o_{i} . X$ from $X_{C}$; and

- $o_{i} . c$ : the number of tuples that need to be fetched and inspected based on the query plan $o_{i} \cdot \mathcal{P}$.

When $o_{i}$ is completed, $o_{i} \cdot \mathcal{P}=\rho_{i}$ and $o_{i} \cdot c=M_{i}$.

Given an SPC query $Q(Z)=\pi_{Z} \sigma_{C}\left(S_{1} \times \ldots \times S_{n}\right)$ that is effectively bounded under $\mathcal{A}$, QPlan returns a set $X_{C}^{\min +}$ of objects such that for $i \in[1, n]$, there exists $o_{i} \in X_{C}^{\min +}$ representing a proof for $X_{C} \mapsto_{\mathcal{I}_{E}}\left(X_{Q}^{i}, M_{i}\right)$.

More specifically, $X_{C}^{\min +}$ is a set of objects such that that $Q$ is effective bounded under $\mathcal{A}$ if and only if for each $i \in[1, n]$, (1) $X_{C}^{i} \subseteq W$ for some $W$ in $\mathcal{X}^{\mathcal{A}}$; and (2) $X_{Q}^{i} \subseteq$ o.X for some object $o$ in $X_{C}^{\min +}$. It has a coverage property: for all 


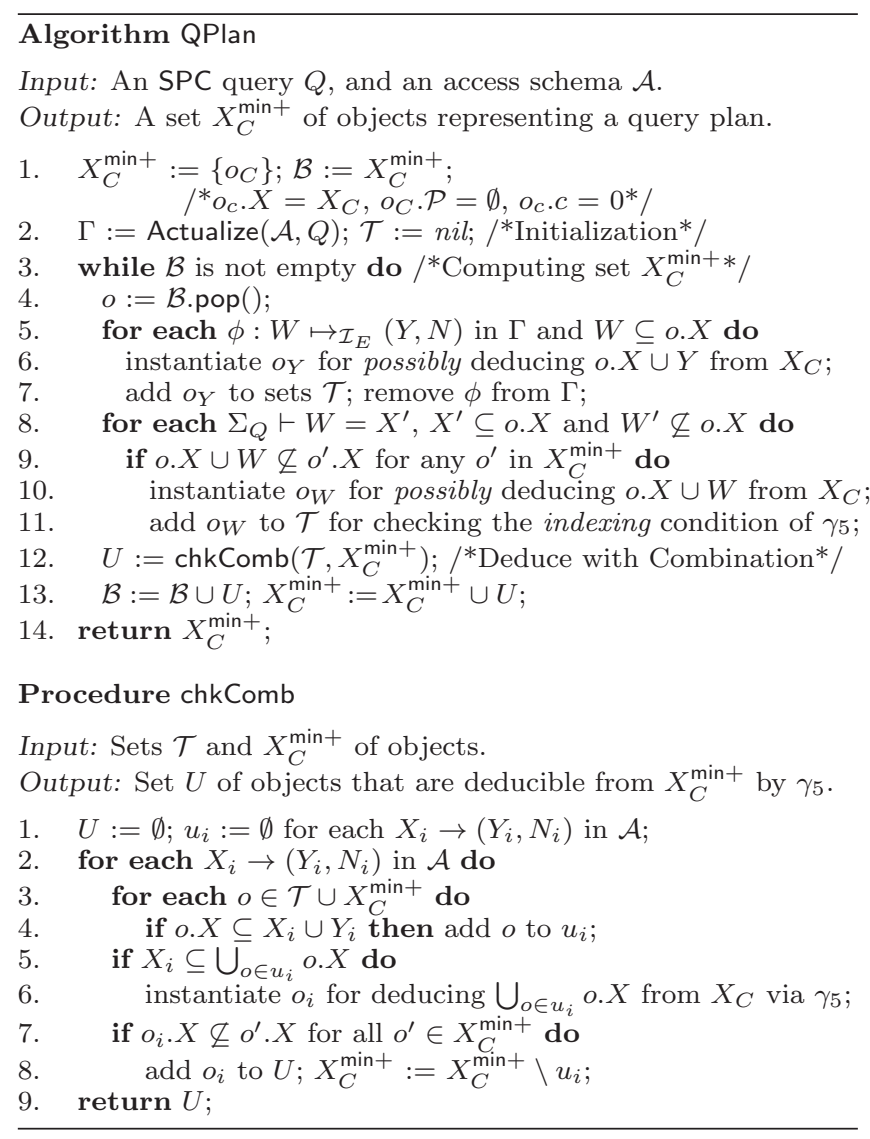

Figure 4: Algorithm QPlan

$Y$, if $X \mapsto \mathcal{I}_{E}(Y, N)$ and $X \subseteq Y$, then there exists some $o \in X_{C}^{\min +}$ such that $Y \subseteq o . X$. These suffice by Theorem 4 .

We use the following notations. (a) A set $S_{2}$ of objects can be deduced from another set $S_{1}$ if there exists a proof from $\bigcup_{o \in S_{1}}$ o.X to $\bigcup_{o \in S_{2}}$ o.X. (b) We use $\gamma_{1}-\gamma_{5}$ to denote the five rules in $\mathcal{I}_{E}$ (Fig. 2), respectively. For instance, $\gamma_{5}$ denotes Combination, and $\gamma_{2}(X \rightarrow(Y, N))$ indicates the application of Actualization with access constraint $X \rightarrow(Y, N)$ in $\mathcal{A}$.

Algorithm QPlan also uses the following structures: (a) a set $\mathcal{B}$ of objects that are in $X_{C}^{\min +}$ but remain to be checked for other objects that can deduced from them, similar to its counterpart used in BCheck (Fig. 3); and (b) a set $\mathcal{T}$ of candidate objects deduced from equality atoms in $\Sigma_{Q}$, which is to be used when Combination rule is applied.

Using these structures, algorithm QPlan works as follows. It first collects in $\Gamma$ all actualized constraints of $\mathcal{A}$ in the same way as BCheck (Fig. 3), and initializes both $X_{C}^{\min +}$ and $\mathcal{B}$ with the set consisting of only one object that represents the proof for $X_{C}$; it sets $\mathcal{T}$ empty (lines 1-2).

After these, QPlan iteratively finds objects that can possibly be deduced from $X_{C}^{\min +}$, by processing objects in $\mathcal{B}$ one by one (lines 3-13). For each object $o$ in $\mathcal{B}$, it finds all possible direct deductions with the actualized constraints, and adds them to $\mathcal{T}$ (lines 5-7). More specifically, if there exists an actualized constraint $\phi: W \mapsto \mathcal{I}_{E}(Y, N)$ in $\Gamma$ and if $W$ is a subset of $o . X$, then $o . X \cup Y$ can possibly be deduced from $X_{C}$ by first deducing $o . X$ using $o . \mathcal{P}$, and then deducing $W$ by using Reflexivity (from $o . X$ ) followed by Transitivity (from $X_{C}$ ), with $o . c=N$, and possibly with Augmentation.
Algorithm QPlan stores these single-step deductions in an object $o_{Y}$ (line 6), and adds it to $\mathcal{T}$ for checking whether o. $X \cup Y$ is indexed in $\mathcal{A}$. It removes $\phi$ from $\Gamma$ (line 7 ).

Intuitively, QPlan expands set $\mathcal{T}$ by including all new candidate objects that can possibly be deduced by $\gamma_{5}$ (i.e., Combination rule), subject to the indexing condition of $\gamma_{5}$ to be checked (lines 8-11). It invokes procedure chkComb to identify combinations of objects in $\mathcal{T}$ to which $\gamma_{5}$ can be applied; chkComb returns a set $U$ of new objects that encode new parameters of $Q$ deduced by $\gamma_{5}$ (line 12 ; see details shortly). The objects of $U$ are added to $X_{C}^{\min +}$ and $\mathcal{B}$ (line 17). The algorithm then proceeds to process the next object in $\mathcal{B}$ in the same way, until $\mathcal{B}$ becomes empty.

After the while loop, QPlan returns $X_{C}^{\min +}$ that contains proofs for each $X_{C} \mapsto_{\mathcal{I}_{E}}\left(X_{Q}^{i}, N_{i}\right)$ (line 14).

Procedure chkComb. Given $\mathcal{T}$ and $X_{C}^{\min +}$, chkComb finds all maximum subsets of $\mathcal{T} \cup X_{C}^{\min +}$ to which rule $\gamma_{5}$ can be applied, to deduce new parameters. More specifically, each subset satisfies the following conditions: (1) the union of their encoded attributes is indexed in $\mathcal{A} ;(2)$ it is maximal, i.e., it cannot be expanded; and (3) no objects in it are already in $X_{C}^{\min +}$. Each of these subsets is encoded by a new object, representing all attributes covered by the subset.

The procedure works as follows. Assume w.l.o.g. that for each object $o$ in $\mathcal{T} \cup X_{C}^{\min +}, o . X$ contains attributes from the same renaming $S_{i}$ only. It associates a set $u_{i}$ with each constraint $X_{i} \rightarrow\left(Y_{i}, A\right)$ in $\mathcal{A}$, initially empty (line 1 ). It collects in $u_{i}$ all objects of $\mathcal{T} \cup X_{C}^{\min +}$ that can be combined using $\gamma_{5}$ and are indexed by $X_{i} \cup Y_{i}$ (lines 2-4). If $X_{i}$ is covered by attributes encoded in the objects of $u_{i}$, then these attributes can be deduced by $\gamma_{5}$ and hence, a new object $o_{i}$ is created to encode them (lines 5-6). If attributes in $o_{i} . X$ are not covered by existing objects in $X_{C}^{\min +}$, then it adds $o_{i}$ to $U$, and removes objects of $u_{i}$ from $X_{C}^{\min +}$ (lines 7-8). The process proceeds until all constraints in $\mathcal{A}$ are checked (line 2). After the loop, it returns set $U$.

Correctness \& Complexity. The correctness of QPlan follows from Theorem 4 and the coverage property of $X_{C}^{\min +}$.

To see that QPlan is in $O\left(|Q|^{2}|\mathcal{A}|^{3}\right)$-time, observe the following. (1) At most $O(|Q \| \mathcal{A}|)$ objects are added to $\mathcal{B}$. This is because each actualized constraint in $\Gamma$ and each equality atom in $\sigma_{C}$ of $Q$ are processed only once; moreover, each equality atom yields at most $O(|\mathcal{A}|)$ objects. (2) The loop (lines 5-11) is executed at most $O(|Q \| \mathcal{A}|)$ times in total. (3) Procedure chkComb is in $O\left(|Q \| \mathcal{A}|^{2}\right)$ time; thus in the entire process, chkComb takes $O\left((|Q||\mathcal{A}|+|\mathcal{A}|) *|Q \| \mathcal{A}|^{2}\right)=$ $O\left(|Q|^{2}|\mathcal{A}|^{3}\right)$ time in total. Indeed, (a) its initialization is in $O(|\mathcal{A}|)$ time; (b) the total time taken by checking indexing (lines 3-4) is $O\left(\left|\mathcal{A} \| \mathcal{T} \cup X_{C}^{\min +}\right|\right)=O\left(|Q \| \mathcal{A}|^{2}\right)$; and (c) checking the conditions of line 5 and line 7 takes $O\left(|Q \| \mathcal{A}|^{2}\right)$ time each. We remark that $|Q|$ and $|\mathcal{A}|$ are typically small in real-life, compared to the size of dataset $D$.

Example 10: We show how QPlan generates a query plan for $Q_{0}$ of Example 1 under access schema $\mathcal{A}_{0}$ of Example 2. Initially, both $X_{C}^{\min +}$ and $\mathcal{B}$ contain an object $o_{C}$ encoding $X_{C}$ such that $o_{C} \cdot X=\left\{\right.$ aid, uid, $\left.\operatorname{tid}_{2}\right\}$ and $o_{C} \cdot \mathcal{P}=$ nil. It then updates $X_{C}^{\min +}$ and $\mathcal{B}$ iteratively. At the beginning, $o_{C}$ is popped off from $\mathcal{B}$. It constructs $o_{1}$ with $o_{1} \cdot X=$ $o_{C} \cdot X \cup\left\{\operatorname{pid}_{1}\right\} o_{1} \cdot \mathcal{P}=\left[\gamma_{1}, \gamma_{2}\left(\right.\right.$ aid $\left.\mapsto_{\mathcal{I}_{E}}\left(\operatorname{pid}_{1}, 1000\right), \gamma_{4}\right]$ and $o_{1} . c=1000 ;$ it puts $o_{1}$ in $\mathcal{T}$. Similarly, it adds $o_{2}$ to $\mathcal{T}$ with $o_{2} \cdot X=o_{C} \cdot X \cup\{\mathrm{fid}\}, o_{2} \cdot \mathcal{P}=\left[\gamma_{1}, \gamma_{2}\right.$ (uid $\mapsto_{\mathcal{I}_{E}}($ fid, 5000$)$, 
$\gamma_{3}$ ] and $o_{2} . c=5000$. After these, it invokes chkComb and finds $U=\left\{o_{1}, o_{2}\right\}$ since $o_{1} . X$ and $o_{2} . X$ are indexed in $\mathcal{A}_{0}$. It replaces $o_{C}$ in $\mathcal{B}$ and $X_{C}^{\min +}$ with $o_{1}$ and $o_{2}$. After that, it pops off $o_{1}$ from $\mathcal{B}$ and finds that equality atom pid $_{1}=$ pid $_{2}$ in $\Sigma_{Q}$ is applicable to $o_{1}$. Thus it adds $o_{3}$ to $\mathcal{T}$ with $o_{3} . X$ $=o_{1} \cdot X \cup\left\{\operatorname{pid}_{2}\right\}, o_{3} \cdot \mathcal{P}=o_{1} \cdot \mathcal{P}$ and $o_{3} \cdot c=1000$. By calling chkComb, $O_{4}$ is deduced using rule $\gamma_{5}$, with $o_{4} . X=o_{3} . X$, $o_{4} \cdot \mathcal{P}=o_{3} \cdot \mathcal{P} \oplus \gamma_{5}(\oplus$ for appending $)$, and $o_{4} \cdot c=1000$.

Note that the parameters of in_album, friends and tagging are covered by $o_{1} . X, o_{2} . X$ and $o_{4} . X$, respectively. Hence $o_{1} \cdot \mathcal{P}, o_{2} \cdot \mathcal{P}$ and $o_{4} \cdot \mathcal{P}$ tell us how to fetch subsets $T_{1}, T_{2}$ and $T_{3}$ from any dataset $D_{0} \models \mathcal{A}_{0}, 7000$ tuples in total. One can verify that $T_{1}, T_{2}$ and $T_{3}$ are precisely those described in Example 1. As shown there, we can fetch $T_{1}, T_{2}$ and $T_{3}$ from $D_{0}$ and compute $Q_{0}\left(D_{0}\right)$ by using these sets only.

\subsection{Minimum $D_{Q}$}

One might be tempted to search for a minimum $D_{Q} \subseteq D$ such that $Q(D)=Q\left(D_{Q}\right)$ under $\mathcal{A}$. More formally, we say that $Q$ is $M$-bounded if for all databases $D$ of schema $R$, there exists a $D_{Q} \subseteq D$ such that $\left|D_{Q}\right| \leq M$ and $Q(D)=$ $Q\left(D_{Q}\right)$. It is effectively $M$-bounded if in addition, $D_{Q}$ can be identified in time independent of $|D|$. These notions were referred to (efficient) scale independence in [20]. The decision problem for finding minimum $D_{Q}$ can be stated as follows:

- Input: $R, Q$ and $\mathcal{A}$, and a natural number $M$.

- Question: Is $Q$ (effectively) $M$-bounded under $\mathcal{A}$ ?

Unfortunately, when $M$ is part of the input, the problem for deciding (effective) boundedness becomes intractable, as opposed to quadratic-time given in Theorems 5 and 6 .

Theorem 8: It is NP-complete to decide whether an SPC query is (a) $M$-bounded or (b) effectively $M$-bounded under an access schema.

We verify this by extending rules in $\mathcal{I}_{B}$ and $\mathcal{I}_{E}[5]$.

\section{EXPERIMENTAL STUDY}

Using real-life and synthetic data, we conducted two sets of experiments to evaluate (1) the effectiveness of our query evaluation approach based on boundedness, and (2) the efficiency of algorithms BCheck, EBCheck, findDP $\mathrm{h}_{\mathrm{h}}$ and QPlan.

Experimental setting. We used three datasets: two reallife (TFACC and MOT) and one synthetic (TPCH).

(1) UK traffic accident (TFACC) was obtained by integrating the Road Safety Data [1], which records information about road accidents that happened in the UK from 1979 to 2005, and the National Public Transport Access Nodes data (NaPTAN) [2], with a fuzzy join on location attributes (latitude, longitude). It has 19 tables with 113 attributes, and over 89.7 million tuples in total. Its size is $21.4 \mathrm{~GB}$.

(2) The Ministry of Transport Test data (MOT [3]) records all MOT tests, including the makes and models of vehicles, odometer reading and reasons for failures, in year 2013. To make the data larger, we joined its 5 tables together. It is of 16.2GB size with 36 attributes and over 55 million tuples.

Synthetic data (TPCH) was generated by using TPC-H dbgen [4]. The dataset consisted of 8 relations. We varied the scale factor from 0.25 to 32 (32 by default) with the size of the data varying from $0.25 \mathrm{~GB}$ to $32 \mathrm{~GB}$.

All of the three datasets were stored in MySQL.
Access schema. We manually extracted 84, 27 and 61 access constraints for TFACC, MOT and TPCH, respectively, by examining the size of their active domains and dependencies of their attributes. For example, on TFACC we had (1) date $\rightarrow($ aid, 610$)$ on relation $R_{\text {acc }}$, indicating that at most 610 accidents happened in the UK in a single day from 1979 to 2005; and (2) aid $\rightarrow$ (vid, 192) on $R_{\mathrm{veh}}$, i.e., at most 192 vehicles were involved in a single accident from 1979 to 2005. For each $X \rightarrow(Y, N)$ extracted, we built index by (a) creating a table by projecting the data on attributes $X \cup Y$, and (b) building an index on $X$ for the new table, using MySQL. We found it easy to extract access constraints from real-life data as above. There are many more such constraints for our datasets, which we did not use in our tests.

SPC queries. We manually designed 45 SPC queries $Q$ on these datasets, 15 for each. The queries vary in the number \#-sel of equality atoms in the selection condition $\sigma_{C}$ of $Q$, which is in the range of $[4,8]$, and the number \#-prod of Cartesian products in $Q$, in the range of $[0,4]$.

Algorithms. We implemented the following algorithms, all in Python: (1) BCheck (Section 4.1) and EBCheck (Section 4.2) for checking boundedness and effective boundedness, respectively; (2) findDP $\mathrm{P}_{\mathrm{h}}($ Section 4.3) to find dominating parameters; (3) QPlan to generate query plans that identify $D_{Q}$ (Section 5.1), (4) evalDQ, a simple algorithm that evaluates effectively bounded SPC queries $Q$ following the query plans generated by QPlan, i.e., fetching $D_{Q}$ from $D$ and evaluating $Q$ on $D_{Q}$, and (5) MySQL, which directly uses MySQL for query evaluation, with all the indices specified in $\mathcal{A}$.

The experiments were conducted on an Amazon EC2 highmemory instance with 17GB memory and 6.5 EC2 compute units. We used MySQL 5.5.35 and MyISAM engine. All the experiments were run 3 times. The average is reported here.

Experimental Results. We next report our findings.

Exp-1: Effectiveness of bounded query evaluation. The first set of experiments evaluated the effectiveness of the bounded query evaluation approach. We first examined the queries generated by using algorithm EBCheck. We found that 35 out of 45 queries are effectively bounded under the access schemas, over $77 \%$. We then evaluated the effectiveness of the query plans generated by QPlan, by comparing the running time of evalDQ with its counterpart of MySQL. The results are reported in Figures 5, on datasets TFACC, MOT and TPCH, by varying $|D|, Q$ and $\|\mathcal{A}\|$ (we use $\|\mathcal{A}\|$ to denote the number of access constraints in $\mathcal{A}$ ). In each of them, we report (a) the average evaluation time (the left $y$-axis), and (b) the size $\left|D_{Q}\right|$ of datasets $D_{Q}$ accessed by evalDQ (the right y-axis). Unless stated otherwise, the tests were conducted on all effectively bounded queries, all access constraints, and full-size datasets by default.

(1) Impact of $|D|$. To evaluate the impact of $|D|$, we varied the size of TFACC and MOT by using scale factors from $2^{-5}$ to 1 , and varied TPCH from $0.25 \mathrm{~GB}$ to $32 \mathrm{~GB}$.

The results are shown in Figures 5(a), 5(e) and 5(i), which tell us the following. (1) The evaluation time of evalDQ is independent of the size of $D$. This verifies our analysis in Section 5. (2) MySQL does not scale well with large $D$. Indeed, evalDQ consistently took $9.3 \mathrm{~s}, 6.2 \mathrm{~s}, 14.7 \mathrm{~s}$ on TFACC, MOT and TPCH, respectively, no matter how large the parts of the datasets were used. In contrast, MySQL took 2024s, 


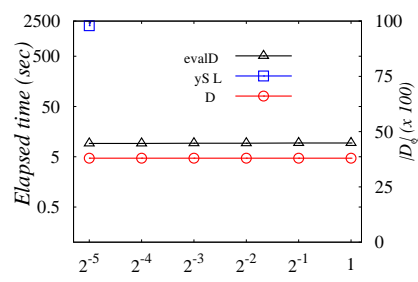

(a) TFACC: varying $|D|(\times 21.4 \mathrm{~GB})$

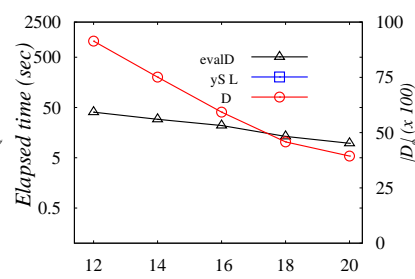

(b) TFACC: varying $\|\mathcal{A}\|$

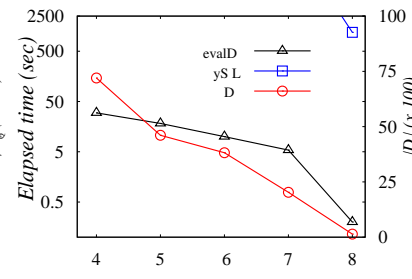

(c) TFACC: varying \#-sel

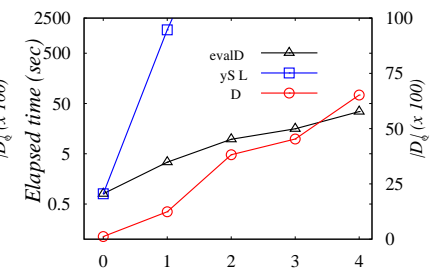

(d) TFACC: varying \#-prod

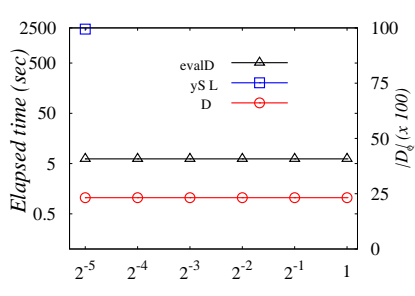

(e) MOT: varying $|D|(\times 16.2 \mathrm{~GB})$

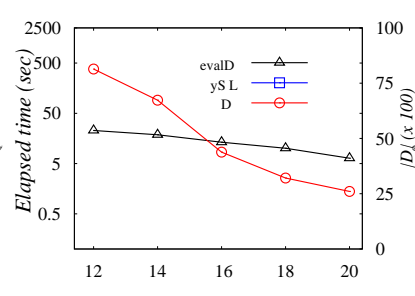

(f) MOT: varying $\|\mathcal{A}\|$

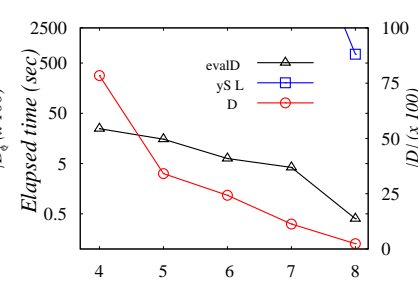

(g) MOT: varying \#-sel

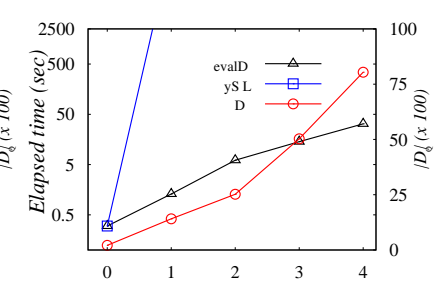

(h) MOT: varying \#-prod

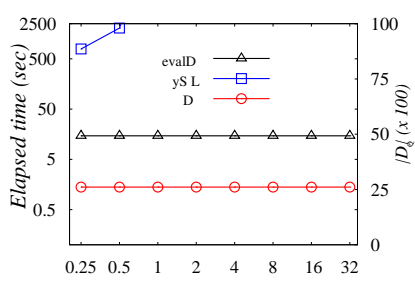

(i) $\mathrm{TPCH}$ : varying $|D|(\mathrm{GB})$

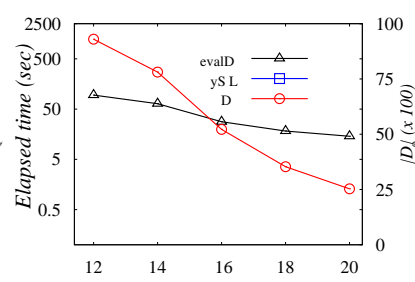

(j) $\mathrm{TPCH}$ : varying $\|\mathcal{A}\|$

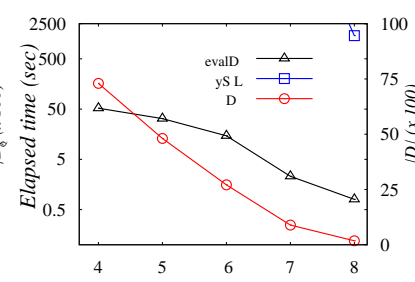

(k) TPCH: varying \#-sel

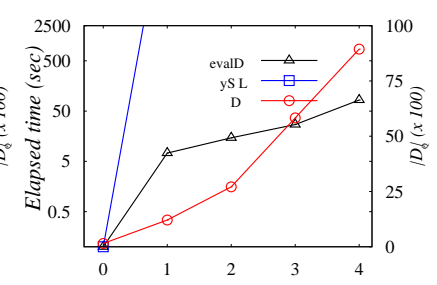

(l) $\mathrm{TPCH}$ : varying \#-prod

Figure 5: Effectiveness of bounded query evaluation

$2367 \mathrm{~s}$ and $2045 \mathrm{~s}$ on subsets of TFACC, MOT and TPCH of sizes $2^{-5} \times 21.4 \mathrm{~GB}, 2^{-5} \times 16.2 \mathrm{~GB}$ and $0.5 \mathrm{~GB}$, respectively, and could not finish its computation within 2500s for all larger subsets. For example, MySQL took longer than 14 hours on the entire TFACC. That is why only a couple of points are reported for MySQL in the figures. That is, even on the smallest subsets we tested, MySQL was $10^{2}$ times slower than evalDQ, and at least $5.4 \times 10^{3}$ time slower on full sized dataset. In fact, the larger the datasets are used, the bigger the gap between MySQL and evaIDQ are. (3) The size $\left|D_{Q}\right|$ of data accessed evalDQ is also independent on $|D|$. Indeed, evalDQ accessed 3800, 2320, 2610 tuples on average, on TFACC, MOT and TPCH, respectively, on all subsets.

(2) Impact of $\|\mathcal{A}\|$. To evaluate the impact of access constraints, we varied $\|\mathcal{A}\|$ from 12 to 20 and tested the queries that are effectively bounded. Accordingly we varied the indices used by MySQL. The results are shown in Figures 5(b), 5(f) and 5(j). The results tell us the following. (1) More access constraints help QPlan get better query plans. For example, when 20 access constraints were used, evalDQ took $9.6 \mathrm{~s}, 6.4 \mathrm{~s}$ and $14.4 \mathrm{~s}$ for queries on TFACC, MOT and TPCH, respectively, as opposed to $40.4 \mathrm{~s}, 22.8 \mathrm{~s}$ and $95 \mathrm{~s}$ with 12 access constraints, although the queries are effectively bounded in both cases. (2) The more constraints are used, the smaller $\left|D_{Q}\right|$ is, as QPlan can find better proofs (query plans) given more options. (3) MySQL did not produce results in any single test within 2500s, no matter whether we used more or less indices embedded in access schemas.

(3) Impact of $Q$. To evaluate the impact of queries, we varied \#-sel of $Q$ from 4 to 8 , and \#-prod of $Q$ from 0 to 4 .

\begin{tabular}{|c|c|c|c|}
\hline \hline Algorithm & TFACC & MOT & TPCH \\
\hline \hline BCheck & $0.8 \mathrm{~s}$ & $0.3 \mathrm{~s}$ & $0.5 \mathrm{~s}$ \\
EBCheck & $0.8 \mathrm{~s}$ & $0.3 \mathrm{~s}$ & $0.5 \mathrm{~s}$ \\
findDP & $0.3 \mathrm{~s}$ & $0.1 \mathrm{~s}$ & $0.2 \mathrm{~s}$ \\
QPlan & $2.1 \mathrm{~s}$ & $0.9 \mathrm{~s}$ & $1.4 \mathrm{~s}$ \\
\hline
\end{tabular}

Table 1: Elapsed Time

We report the average evaluation time of evalDQ and the size $\left|D_{Q}\right|$ for all queries with the same \#-sel or \#-prod, in Figures 5(c), 5(g) and 5(k), and Figures 5(d), 5(h) and 5(l), respectively. They tell us the following. (1) The complexity of $Q$ has impacts on the quality of query plans generated by QPlan. The larger \#-sel or the smaller \#-prod is, the better the evaluation time of evalDQ and the size $\left|D_{Q}\right|$ of data accessed by evalDQ are, as expected. (2) Algorithm evalDQ scales well with \#-sel and \#-prod. It finds answers in all cases within 90s, on the three full datasets. (3) MySQL is indifferent to \#-sel. But it is sensitive to \#-prod: it is as fast as evalDQ when \#-prod =0, i.e., when there is no Cartesian product at all; but it cannot stop within 2500s for queries even with 1 Cartesian product, except one case of TFACC.

To understand the gap in performance between MySQL and ours, we examined the system logs and found the following. Given an access constraint $X \rightarrow(Y, N)$ on a relation $R$, evalDQ fetched only relevant $(X, Y)$ attribute values; in contrast, MySQL fetched entire tuples with irrelevant attributes of $R$, even with the index on $X$; this led to duplicated $(X, Y)$ values, and the duplications got rapidly inflated by Cartesian product; hence the gap in performance.

Exp-2: Efficiency. The second set of experiments evaluated the efficiency of our algorithms BCheck, EBCheck, findDP $\mathrm{h}_{\mathrm{h}}$ and QPlan on queries and access schemas for each 


\begin{tabular}{|c|c|c|}
\hline \hline Problem & $M$ is not predefined & $M$ is part of input \\
\hline \hline $\operatorname{Bnd}(Q, \mathcal{A})$ & $O(|Q|(|\mathcal{A}|+|Q|))($ Th 5$)$ & NP-complete (Th 8) \\
\hline $\operatorname{EBnd}(Q, \mathcal{A})$ & $O(|Q|(|\mathcal{A}|+|Q|))($ Th 6$)$ & NP-complete $($ Th 8$)$ \\
\hline $\operatorname{DP}(Q, \mathcal{A})$ & NP-complete (Th 7) & NP-complete [5] \\
\hline $\operatorname{MDP}(Q, \mathcal{A})$ & NPO-complete (Th 7) & NPO-complete [5] \\
\hline
\end{tabular}

Table 2: Complexity bounds

of TFACC, MOT and TPCH. We used all access constraints, and report in Table 1 the longest elapsed time of each algorithm on all queries for each dataset. These results verify that all of our algorithms are efficient: for all queries, all of our algorithms took no more than 2.1 seconds, even QPlan, the one with the highest complexity (see Section 5). These confirm our complexity analyses of these algorithms.

Summary. From the experimental results we find the following. (1) The notion of effective boundedness is practical. It is rather easy to find sufficiently many access constraints in real-life data, and many practical queries are actually effectively bounded. (2) The bounded query evaluation approach allows us to query big data. Its evaluation time and amount of data accessed are independent of the size of the underlying dataset. For example, on a real-life dataset of 21.4GB, evalDQ finds answers to queries in 9.3 seconds by accessing no more than 3800 tuples on average. In contrast, MySQL is unable to get answers within 2500 seconds in almost all of the cases except for extremely restricted queries (without Cartesian products). Even on a dataset of $2^{-4} \times 21.4 \mathrm{~GB}(1.3 \mathrm{~GB})$, it took longer than 3 hours. The gap between evalDQ and MySQL is more substantial on larger datasets. (3) Our algorithms are efficient: they are able to check (effective) boundedness, identify dominating parameters, and generate query plans in 2.1 seconds for queries defined on large schemas and a variety of access constraints.

\section{CONCLUSION}

We have studied (effective) boundedness for SPC, a class of queries that are widely used in practice (cf. [23]). We have investigated fundamental problems to characterize what SPC query $Q$ can be evaluated under an access schema $\mathcal{A}$, and to make $Q$ effectively bounded under $\mathcal{A}$ by identifying a minimum set of parameters to instantiate. We have established their complexity bounds, as summarized in Table 2. We have also developed efficient (heuristic) algorithms to make practical use of effective boundedness. Our experimental results have verified that effective boundedness yields a promising approach to querying big data.

The study is still in its infancy. (1) It is undecidable to decide whether an $\mathcal{R} \mathcal{A}$ query is (effectively) bounded [20]. Nonetheless, we can still find efficient heuristic algorithms to check the effective boundedness of $\mathcal{R} \mathcal{A}$. (2) Given a set of parameterized queries, we want to study how to build an optimal access schema under which the queries are effectively bounded. (3) When a query is not effectively bounded, it may be effectively bounded incrementally or using views. A preliminary study of these issues has been reported in [11, 20]. However, effective algorithms remain to be developed.

Acknowledgment. Cao, Fan and $\mathrm{Yu}$ are supported in part by 973 Programs 2014CB340302 and 2012CB316200, NSFC 61133002, Guangdong Innovative Research Team Program 2011D005, Shenzhen Peacock Program 1105100030834361, China, and EPSRC EP/J015377/1, UK. Cao is also supported by the Fundamental Research Funds for the Central Universities YWF-14-RSC-018, China.

\section{REFERENCES}

[1] http://data.gov.uk/dataset/road-accidents-safety-data.

[2] http://data.gov.uk/dataset/naptan.

[3] http://data.gov.uk/dataset/anonymised_mot_test.

[4] http://www.tpc.org/tpch/.

[5] full. http://homepages.inf.ed.ac.uk/s1165433/bounded.pdf.

[6] S. Abiteboul, R. Hull, and V. Vianu. Foundations of Databases. Addison-Wesley, 1995.

[7] S. Acharya, P. B. Gibbons, V. Poosala, and S. Ramaswamy. Join synopses for approximate query answering. In SIGMOD, 1999.

[8] S. Agarwal, B. Mozafari, A. Panda, H. Milner, S. Madden, and I. Stoica. BlinkDB: queries with bounded errors and bounded response times on very large data. In EuroSys, 2013.

[9] M. Armbrust, K. Curtis, T. Kraska, A. Fox, M. J. Franklin, and D. A. Patterson. PIQL: Success-tolerant query processing in the cloud. In $V L D B, 2011$.

[10] M. Armbrust, A. Fox, D. A. Patterson, N. Lanham, B. Trushkowsky, J. Trutna, and H. Oh. SCADS: Scale-independent storage for social computing applications. In $C I D R, 2009$.

[11] M. Armbrust, E. Liang, T. Kraska, A. Fox, M. J. Franklin, and D. Patterson. Generalized scale independence through incremental precomputation. In SIGMOD, 2013.

[12] A. Atserias, M. Grohe, and D. Marx. Size bounds and query plans for relational joins. In FOCS, 2008.

[13] G. Ausiello. Complexity and approximation: Combinatorial optimization problems and their approximability properties. Springer, 1999.

[14] B. Babcock, S. Chaudhuri, and G. Das. Dynamic sample selection for approximate query processing. In $S I G M O D$, 2003.

[15] V. Bárány, M. Benedikt, and P. Bourhis. Access patterns and integrity constraints revisited. In ICDT, 2013.

[16] A. Deutsch, B. Ludäscher, and A. Nash. Rewriting queries using views with access patterns under integrity constraints. TCS, 371(3), 2007.

[17] M. Ester, H.-P. Kriegel, J. Sander, and X. Xu. A density-based algorithm for discovering clusters in large spatial databases with noise. In $K D D, 1996$.

[18] Facebook. http://newsroom.fb.com.

[19] Facebook. Constraints on the number of photos, friends and tags. https://www.facebook.com/help \{/227794810567981, /community/question/?id=364005057055660\}, 2014.

[20] W. Fan, F. Geerts, and L. Libkin. On scale independence for querying big data. In PODS, 2014.

[21] W. Fan, F. Geerts, and F. Neven. Making queries tractable on big data with preprocessing. In VLDB, 2013.

[22] M. N. Garofalakis and P. B. Gibbons. Wavelet synopses with error guarantees. In SIGMOD, 2004.

[23] G. Gottlob, S. T. Lee, and G. Valiant. Size and treewidth bounds for conjunctive queries. In PODS, 2009.

[24] Y. E. Ioannidis and V. Poosala. Histogram-based approximation of set-valued query-answers. In $V L D B, 1999$.

[25] H. V. Jagadish, N. Koudas, S. Muthukrishnan, V. Poosala, K. C. Sevcik, and T. Suel. Optimal histograms with quality guarantees. In $V L D B, 2009$.

[26] P. C. Kanellakis. On the computational complexity of cardinality constraints in relational databases. Inf. Process. Lett., 11(2):98-101, 1980.

[27] L. Kaufman and P. J. Rousseeuw. Finding Groups in Data: An Introduction to Cluster Analysis. John Wiley, 1990.

[28] C. Li. Computing complete answers to queries in the presence of limited access patterns. VLDB J., 12(3), 2003.

[29] P. Rösch and W. Lehner. Sample synopses for approximate answering of group-by queries. In EDBT, 2009. 